TOPICAL REVIEW

\title{
Application of the density matrix renormalization group method to finite temperatures and two-dimensional systems
}

\author{
Naokazu Shibata \\ Department of Basic Science, University of Tokyo, Komaba, Tokyo 153-8902 Japan
}

\begin{abstract}
The density matrix renormalization group (DMRG) method and its applications to finite temperatures and two-dimensional systems are reviewed. The basic idea of the original DMRG method, which allows precise study of the ground state properties and low-energy excitations, is presented for models which include long-range interactions. The DMRG scheme is then applied to the diagonalization of the quantum transfer matrix for one-dimensional systems, and a reliable algorithm at finite temperatures is formulated. Dynamic correlation functions at finite temperatures are calculated from the eigenvectors of the quantum transfer matrix with analytical continuation to the real frequency axis. An application of the DMRG method to twodimensional quantum systems in a magnetic field is demonstrated and reliable results for quantum Hall systems are presented.
\end{abstract}




\section{Introduction}

Numerical calculations are now commonly used in various studies in physics. The recent remarkable advance in computer technology and the development of efficient algorithms provide us with a growing opportunity to resolve behaviors of various interacting systems leading us to a new understanding of natural phenomena. In condensed matter physics, one of the recent progresses has been made by the density matrix renormalization group (DMRG) method, which was developed by Steven White in 1992 [1, 2]. This method is a type of variational method combined with a real space renormalization group method, which enables us to obtain the ground-state wavefunction of large-size systems with controlled high accuracy. The DMRG method has excellent features compared with other standard numerical methods. In contrast to the quantum Monte Carlo method, the DMRG method is free from statistical errors and the negative sign problem which hampers convergence of physical quantities at low temperatures. Furthermore, the essentially exact ground state of large systems extending the limitation of the exact diagonalization method is obtained within a restricted number of basis states. The truncation error caused by the restriction of basis states is systematically controlled by the density matrix calculated from the ground-state wavefunction and the obtained results are easily improved by increasing the number of basis states retained in the system.

The ground-state properties of frustrated quantum spin systems and the strongly correlated electron systems in one dimension have been extensively studied by the DMRG method. Our understanding of these systems was greatly advanced and such successful calculations on one-dimensional quantum systems have promoted many applications to other problems. In 1995, Nishino applied the DMRG method to the transfer matrix and he calculated the partition function of two-dimensional classical systems at finite temperatures [3. In 1996, Bursill et al applied this method to the quantum transfer matrix (QTM) for $S=1 / 2 \mathrm{XY}$ spin chains, and calculated thermodynamic quantities at finite temperatures. However, they used symmetric projection $\left|V^{R}\right\rangle\left\langle V^{R}\right|$ for the calculation of the density matrix and reliable results were obtained only down to $T / J=0.2$ [4]. Correct calculations were done for $S=1 / 2$ Heisenberg spin chains by Wang and Xiang [5], and Shibata [6] in 1997, and they obtained accurate results down to $T / J=0.01$. The thermodynamic quantities and spin correlation length of the Heisenberg chains with $S=1 / 2,1$, and $3 / 2$ were systematically calculated by Xiang [7]. The stability of this method for fermion systems was shown in the calculation of thermodynamic quantities of the one-dimensional Kondo lattice model, which is a canonical model of heavy fermion systems [8]. In 1999 the reliability of the method was also shown for frustrated quantum spin chains, $t-J$ ladders and the Kondo lattice model away from half-filling [9, 10, 11, where the negative sign problem arises in the quantum Monte Carlo simulations. Dynamic correlation functions and one-particle Green's functions in the Kondo lattice model were calculated at finite temperatures, and the temperature-induced gap formation in the Kondo insulators was 
clarified [12]. There has been a review on the DMRG calculations of thermodynamic and dynamic quantities of the Kondo lattice model [13. Autocorrelations in quantum spin chains were systematically studied by Naef et al and its reliability was discussed [14. The thermodynamic quantities of a spin-Peierls system of $\mathrm{CuGeO}_{3}$ was studied by Klümper et al [15]. Rommer and Eggert applied the finite-temperature DMRG method to impurity Kondo problems, and their results were shown to be consistent with the predictions of field theory calculations [16. The quasi-particle density of states and the dynamic spin and charge correlation functions of the doped one-dimensional Kondo insulators were calculated and their doping dependence was clarified [17. In 2000, Naef and Wang [18] studied the nuclear spin relaxation rate $1 / T_{1}$ in the twoleg spin ladder and compared the results to theoretical predictions and experimental measures. Maeshima and Okunishi [19] calculated thermodynamic quantities of frustrated quantum spin chains under a magnetic field and studied behaviors near the critical fields in the ground-state magnetization process. The magnetization process of the Heisenberg spin ladder was analyzed by Wang and $\mathrm{Yu}$ [20], and they determined the phase diagram consisting of disordered spin liquid, the Luttinger liquid, spin-polarized phases, and a classical regime. Ammon and Imada 21, 22, 23] systematically studied the low-temperature properties of doped $S=1$ spin chains and clarified behaviors of various correlation functions. In 2001, the thermodynamic properties of the $S=1 / 2$ Heisenberg chain with a staggered Dzyaloshinsky-Moriya interaction were studied and anomalous behaviors in $\mathrm{Yb}_{4} \mathrm{As}_{3}$ were explained [24, 25]. The thermodynamic properties of the $t-J$ chain were studied by Sirker and Klümper, and the crossover behavior to Tomonaga-Luttinger liquid was shown [26, 27]. Maruyama et al [28] studied properties of a non-magnetic impurity in Kondo insulators. Recently, Sirker and Khaliullin [29] studied dimerization in a one-dimensional spin-orbital model with spins $S=1$.

The application of the DMRG method to two-dimensional quantum systems is currently the most challenging subject and many algorithms have been proposed. Most of them use mappings on to effective one-dimensional models with long-range interactions, and the standard DMRG method is applied to the effective one-dimensional systems. In 1996, White 30 applied the DMRG method to a two-dimensional frustrated quantum spin model for $\mathrm{CaV}_{4} \mathrm{O}_{9}$ and showed the existence of the spin gap. In 1998, White and Scalapino [31] studied the two-dimensional $t$ - $J$ model with a hole doping and found a striped phase. They also studied the competition between stripes and pairing in an $n$-leg $t-t^{\prime}-J$ model [32], and made a critical analysis on the phase separation and stripe formation in the two-dimensional $t$ - $J$ model [33]. In 2001, Shibata and Yoshioka [34, 35] applied the DMRG method to quantum Hall systems and the ground state phase diagram of two-dimensional electrons in a high Landau level was determined. Xiang et al [36] proposed an efficient mapping on to a one-dimensional system which retains the topological characteristics of two-dimensional lattices. They applied this method to the $S=1 / 2$ Heisenberg model on both square and triangular lattices. In 2003, the ground state phase diagram of two-dimensional electrons in the lowest and the second lowest Landau levels were determined and the existence of various quantum liquids and charge 


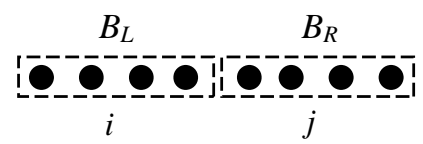

Figure 1. The system divided into two blocks $B_{L}$ and $B_{R}$, whose basis states are represented by indices $i$ and $j$. The solid circles represent local orbitals or quantum spins.

ordered states, such as Laughlin state and the Wigner crystal, was confirmed with new stripe states [37.

Another approach to higher dimensions was proposed by Xiang in 1996 in which the Hamiltonian is renormalized in momentum space [38, 39]. This method is clearly efficient for weakly interacting systems. The DMRG method has also been applied to classical systems in higher dimensions [3]. In 1996 the DMRG method was used to renormalize the corner transfer matrix and the corner transfer matrix renormalization group (CTMRG) method was formulated [40]. This method was extended to threedimensional classical models in 1998 [41]. Recently the CTMRG method was extended to study one-dimensional stochastic models 42. The DMRG algorithm has also been used to optimize tensor product states in three-dimensional classical systems, and this method was applied to two-dimensional quantum spin systems at finite temperatures 43. There has been an analytical study on the spectra of the density matrices used in the DMRG calculations for two-dimensional systems [4]. Several topics of the DMRG calculations are summarized in a book published in 1998 [45].

In this topical review, the applications of the DMRG method to finite temperatures and two-dimensional quantum systems are reviewed with various techniques in the calculation. We first briefly summarize the basic idea of the DMRG method and explain how we treat long range interactions. We then apply the DMRG scheme to the QTM and formulate an algorithm for the calculations of thermodynamic quantities and dynamic correlation functions at finite temperatures. We also apply the DMRG algorithm to twodimensional quantum systems in a magnetic field and calculate various ground states realized in quantum Hall systems.

\section{Zero temperature DMRG}

A numerical study of quantum many-body problems requires the handling of exponentially increasing basis states of the system. For example, when we represent the Hamiltonian of two-spin system with $S=1 / 2$, four basis states, $|\uparrow, \uparrow>,| \uparrow, \downarrow>$, $\mid \downarrow, \uparrow>$, and $\mid \downarrow, \downarrow>$ are needed. As is easily expected, the dimension of the Hamiltonian

exponentially increases as $2^{N}$ with the increase of the number of spins $N$. This exponential dependence prevents exact numerical diagonalizations for more than 30 spins in usual cases. The DMRG method is designed to diagonalize the Hamiltonian of large-size systems extending this limitation of the exact diagonalization by restricting the basis states. Here we briefly review how the DMRG method restricts the basis states 
with controlled accuracy.

\subsection{Restriction of basis states}

Let us consider a system consisting of two blocks, $B_{L}$ and $B_{R}$ (see figure 1), and represent the ground-state wavefunction $|\Psi\rangle$ using the basis states of the blocks described by $|i\rangle$ and $|j\rangle$ :

$$
|\Psi\rangle=\sum_{i, j} \Psi_{i j}|i\rangle|j\rangle
$$

Here we define the following density matrix $\rho$ for the block $B_{L}$, whose basis states are represented by $|i\rangle$ :

$$
\rho_{i i^{\prime}}^{L}=\sum_{j} \Psi_{i j}^{*} \Psi_{i^{\prime} j}
$$

The norm of the wavefunction is then written by

$$
\langle\Psi \mid \Psi\rangle=\sum_{i, j} \Psi_{i j}^{*} \Psi_{i j}=\sum_{i} \rho_{i i}^{L}=\operatorname{Tr} \rho^{L} .
$$

The question is how we reduce the number of basis states in the block $B_{L}$ while keeping $\operatorname{Tr} \rho^{L}$ as much as possible. Since $\operatorname{Tr} \rho^{L}$ is equivalent to the sum of the eigenvalues of $\rho^{L}$, we first solve the eigenvalue equation

$$
w^{\alpha} v_{i}^{\alpha}=\sum_{i^{\prime}} \rho_{i i^{\prime}}^{L} v_{i^{\prime}}^{\alpha}
$$

and transform the basis states from $|i\rangle$ to $|\alpha\rangle$ which is defined by

$$
|\alpha\rangle=\sum_{i} v_{i}^{\alpha}|i\rangle
$$

Since $\rho^{L}$ is Hermitian, the basis states $|\alpha\rangle$ satisfy the orthogonality

$$
\left\langle\alpha \mid \alpha^{\prime}\right\rangle=\sum_{i}\left(v_{i}^{\alpha}\right)^{*} v_{i}^{\alpha^{\prime}}=\delta_{\alpha \alpha^{\prime}}
$$

After this transformation, the density matrix becomes diagonal and the norm of the wavefunction is written by

$$
\langle\Psi \mid \Psi\rangle=\operatorname{Tr} \rho^{L}=\sum_{\alpha} w^{\alpha} .
$$

This result clearly shows that the norm is efficiently preserved by keeping only eigenvectors whose eigenvalues $w^{\alpha}$ are large. Thus the optimal $m$ basis states are obtained from the eigenstates of the $m$ largest eigenvalues of the density matrix. Since

$\rho^{L}$ is the product of $\Psi_{i j}$ and its Hermitian conjugate $\Psi_{i j}^{*}$, the eigenvalues $w^{\alpha}$ are all positive and we can sort $w^{\alpha}$ as

$$
w^{1} \geq w^{2} \geq w^{3} \geq \ldots \geq w^{N} \geq 0
$$

where $N$ is the number of basis states in $B_{L}$. The truncation error, $\operatorname{Er}(m)$, of the wavefunction represented by $m$ basis states in $B_{L}$ is then given by

$$
\operatorname{Er}(m)=\sum_{\alpha=m+1}^{N} w^{\alpha}=1-\sum_{\alpha=1}^{m} w^{\alpha} .
$$




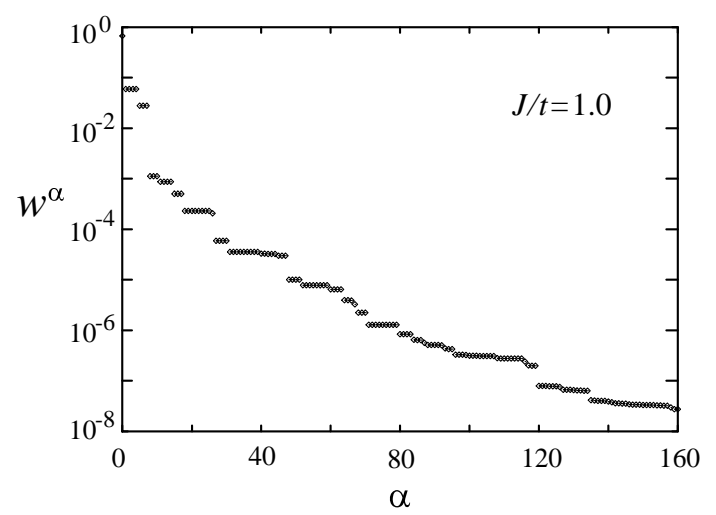

Figure 2. Eigenvalues of the density matrix obtained in the one-dimensional Kondo lattice model. The number of conduction electrons and localized spins are both 32 . The electrons and the spins are coupled through on-site antiferromagnetic exchange coupling $J . t$ is the hopping integral.

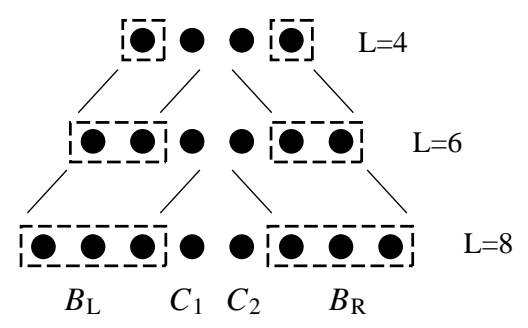

Figure 3. Schematic diagram for infinite system algorithm of the DMRG.

Here we have used $\langle\Psi \mid \Psi\rangle=\sum_{\alpha=1}^{N} w^{\alpha}=1$. Thus the truncation error depends on the distribution of the eigenvalues of the density matrix, and high accuracy is obtained when $w^{\alpha}$ decays rapidly with increasing $\alpha$. A typical example of $w^{\alpha}$ obtained for the onedimensional Kondo lattice model is shown in figure 2, Even though this model consists of conduction electrons and localized spins, the accuracy of $10^{-7}$ is attained by keeping only 150 states in each block. Accurate results in the DMRG calculations are obtained when $w^{\alpha}$ decays almost exponentially as shown in figure 2 .

\subsection{Infinite system algorithm}

Here we describe how we obtain the Hamiltonian of a large system within a restricted number of basis states. In the infinite system algorithm of the DMRG method[1, we start from a small system and iteratively extend the system by adding new sites between the two blocks, as schematically shown in figure 3. At each extension of the blocks we restrict the basis states using the density matrix defined in section 2.1. In the following, we describe the algorithm of the DMRG method applied to one-dimensional quantum systems which have long-range interactions.

We usually start the calculation from a system consisting of four single sites with 
open boundary conditions. The left and right blocks contain the single site at one end of the system, as shown in figure 3. The Hamiltonian is written by

$$
\begin{aligned}
H= & H^{B_{L}}+H^{C_{1}}+H^{C_{2}}+H^{B_{R}} \\
& +H^{B_{L}-C_{1}}+H^{C_{1}-C_{2}}+H^{C_{2}-B_{R}} \\
& +H^{B_{L}-C_{2}}+H^{C_{1}-B_{R}}+H^{B_{L}-B_{R}}
\end{aligned}
$$

where $H^{B_{L}}$ and $H^{B_{R}}$ contains operators represented by only the basis states of the left and right blocks, respectively, and $H^{C_{1}}$ and $H^{C_{2}}$ are the single-site Hamiltonian between the two blocks. The interaction and hopping terms between different blocks and single sites are represented by $H^{B_{L}-C_{1}}+H^{C_{1}-C_{2}}+H^{C_{2}-B_{R}}$ and $H^{B_{L}-C_{2}}+H^{C_{1}-B_{R}}+H^{B_{L}-B_{R}}$, where the former three terms describe the nearest-neighbor interactions and hoppings, and the latter three terms represent next-nearest-neighbor and further long-range interactions and hoppings. In order to avoid complexity we have assumed that each inter-site interaction and hopping term is written by operators at two different sites, and there are no multi-site interactions such as $\mathbf{S}_{l} \cdot\left(\mathbf{S}_{m} \times \mathbf{S}_{n}\right)$ with $l \neq m \neq n$. The Heisenberg model with nearest-neighbor interaction $J_{1}$ and next-nearest-neighbor interaction $J_{2}$ in magnetic field $h^{z}$ is written by $H^{B_{L}}=h^{z} S_{1}^{z}, H^{C_{1}}=h^{z} S_{2}^{z}, H^{C_{2}}=h^{z} S_{3}^{z}, H^{B_{R}}=h^{z} S_{4}^{z}$, $H^{B_{L}-C_{1}}=J_{1} \mathbf{S}_{1} \cdot \mathbf{S}_{2}, H^{C_{1}-C_{2}}=J_{1} \mathbf{S}_{2} \cdot \mathbf{S}_{3}, H^{C_{2}-B_{R}}=J_{1} \mathbf{S}_{3} \cdot \mathbf{S}_{4}, H^{B_{L}-C_{2}}=J_{2} \mathbf{S}_{1} \cdot \mathbf{S}_{3}$, $H^{C_{1}-B_{R}}=J_{2} \mathbf{S}_{2} \cdot \mathbf{S}_{4}$, and $H^{B_{L}-B_{R}}=0$.

We then calculate the ground state-wavefunction $|\Psi\rangle$ by diagonalizing the above Hamiltonian. The obtained wavefunction is represented in the basis states of the blocks and single sites as

$$
|\Psi\rangle=\sum_{i_{L} i_{1} i_{2} i_{R}} \Psi_{i_{L} i_{1} i_{2} i_{R}}\left|i_{L}\right\rangle\left|i_{1}\right\rangle\left|i_{2}\right\rangle\left|i_{R}\right\rangle
$$

where $\left|i_{L}\right\rangle$ and $\left|i_{R}\right\rangle$ are the basis states of $B_{L}$ and $B_{R}$, and $\left|i_{1}\right\rangle$ and $\left|i_{2}\right\rangle$ are the basis states of $C_{1}$ and $C_{2}$. We next extend the blocks by including the neighboring single site. The basis states of the extended blocks $B_{L} \otimes C_{1}$ and $B_{R} \otimes C_{2}$ are represented by the product space of the original block and the single site, $\left|i_{L}\right\rangle\left|i_{1}\right\rangle$ and $\left|i_{R}\right\rangle\left|i_{2}\right\rangle$. We then restrict the basis states of the extended blocks. For this purpose we calculate the density matrices for the extended blocks, $\rho^{L}$ and $\rho^{R}$ defined in section 2.1

$$
\begin{aligned}
& \rho_{i_{L} i_{1}, i_{L}^{\prime} i_{1}^{\prime}}^{L}=\sum_{i_{R} i_{2}} \Psi_{i_{L} i_{1} i_{2} i_{R}} \Psi_{i_{L}^{\prime} i_{1}^{\prime} i_{2} i_{R}}^{*} \\
& \rho_{i_{R} i_{2}, i_{R}^{\prime} i_{2}^{\prime}}^{R}=\sum_{i_{L} i_{1}} \Psi_{i_{L} i_{1} i_{2} i_{R}} \Psi_{i_{L} i_{1} i_{2}^{\prime} i_{R}^{\prime}}^{*} .
\end{aligned}
$$

We diagonalize the density matrices to obtain the eigenvectors $\vec{v}^{\alpha}$ and the corresponding eigenvalues $w^{\alpha}$ which satisfy

$$
\begin{aligned}
& w^{\alpha_{L}} v_{i_{L} i_{1}}^{\alpha_{L}}=\sum_{i_{L}^{\prime} i_{1}^{\prime}} \rho_{i_{L} i_{1}, i_{L}^{\prime} i_{1}^{\prime}}^{L} v_{i_{L}^{\prime} i_{1}^{\prime}}^{\alpha_{L}} \\
& w^{\alpha_{R}} v_{i_{R} i_{2}}^{\alpha_{R}}=\sum_{i_{R}^{\prime} i_{2}^{\prime}} \rho_{i_{R} i_{2}, i_{R}^{\prime} i_{2}^{\prime}}^{R} v_{i_{R}^{\prime} i_{2}^{\prime}}^{\alpha_{R}} .
\end{aligned}
$$


The new basis states of the extended blocks are obtained from the eigenvectors of the $m$ largest eigenvalues $\left|\alpha_{L}\right\rangle$ and $\left|\alpha_{R}\right\rangle$ defined by

$$
\begin{aligned}
\left|\alpha_{L}\right\rangle & =\sum_{i_{L} i_{1}} v_{i_{L} i_{1}}^{\alpha_{L}}\left|i_{L}\right\rangle\left|i_{1}\right\rangle \\
\left|\alpha_{R}\right\rangle & =\sum_{i_{R} i_{2}} v_{i_{R} i_{2}}^{\alpha_{R}}\left|i_{R}\right\rangle\left|i_{2}\right\rangle .
\end{aligned}
$$

We then represent all operators using these new basis states. The spin operator at the first site $\left(S_{1}^{z}\right)_{i_{L} i_{L}^{\prime}}$ in the left block $B_{L}$ is given by

$$
\left(S_{1}^{z}\right)_{\alpha_{L} \alpha_{L}{ }^{\prime}}=\sum_{i_{L} i_{L} i_{1}} v_{i_{L} i_{1}}^{\alpha_{L}}\left(v_{i_{L}{ }^{\prime} i_{1}}^{\alpha_{\alpha^{\prime}}}\right)^{*}\left(S_{1}^{z}\right)_{i_{L} i_{L}{ }^{\prime}}
$$

Similarly, the spin operators at the second site $\left(S_{2}^{z}\right)_{i_{1} i_{1}^{\prime}}$ which has been included in the new block $\left(B_{L}\right)$ new is represented by

$$
\left(S_{2}^{z}\right)_{\alpha_{L} \alpha_{L}{ }^{\prime}}=\sum_{i_{L} i_{1} i_{1}{ }^{\prime}} v_{i_{L} i_{1}}^{\alpha_{L}}\left(v_{i_{L} i_{1}{ }^{\prime}}^{\alpha_{L}^{\prime}}\right)^{*}\left(S_{2}^{z}\right)_{i_{1} i_{1}{ }^{\prime}}
$$

The block Hamiltonian $\left(H^{B_{L}}\right)_{\text {new }}=H^{B_{L}}+H^{C_{1}}+H^{B_{L}-C_{1}}$ and the interaction between the new block and the single site $\left(H^{B_{L}-C_{1}}\right)_{\text {new }}=H^{C_{1}-C_{2}}+H^{B_{L}-C_{2}}$ are obtained by

$$
\begin{aligned}
& \left(H_{\alpha_{L} \alpha_{L}^{\prime}}^{B_{L}}\right)_{\text {new }}=\sum_{i_{L} i_{L^{\prime} i_{1} i_{1}{ }^{\prime}}} v_{i_{L} i_{1}}^{\alpha_{L}}\left(v_{i_{L}^{\prime} i_{1}^{\prime}}^{\alpha_{L}^{\prime}}\right)^{*}\left\{H_{i_{L} i_{L}^{\prime}}^{B_{L}} \delta_{i_{1} i_{1}^{\prime}}+H_{i_{1} i_{1}^{\prime}}^{C_{1}} \delta_{i_{L} i_{L}^{\prime}}+H_{i_{L} i_{1} i_{L}^{\prime} i_{1}^{\prime}}^{B_{L}-C_{1}}\right\}
\end{aligned}
$$

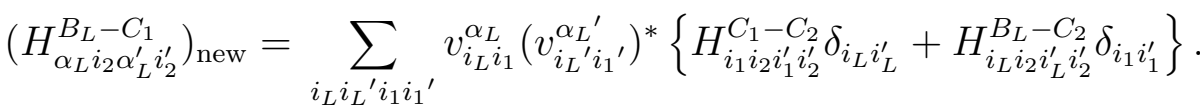

Similarly, $\left(H^{B_{R}}\right)_{\text {new }}$ and $\left(H^{C_{2}-B_{R}}\right)_{\text {new }}$ are given by

$$
\begin{aligned}
& \left(H_{\alpha_{R} \alpha_{R}^{\prime}}^{B_{R}}\right)_{\text {new }}=\sum_{i_{R} i_{R}{ }^{\prime} i_{2} i_{2}{ }^{\prime}} v_{i_{R} i_{2}}^{\alpha_{R}}\left(v_{i_{R}{ }^{\prime} i_{2}{ }^{\prime}}^{\alpha_{\alpha^{\prime}}{ }^{\prime}}{ }^{*}\left\{H_{i_{R} i_{R}^{\prime}}^{B_{R}} \delta_{i_{2} i_{2}^{\prime}}+H_{i_{2} i_{2}^{\prime}}^{C_{2}} \delta_{i_{R} i_{R}^{\prime}}+H_{i_{R} i_{2} i_{R} i_{2}^{\prime}}^{C_{2}-B_{R}}\right\}\right. \\
& \left(H_{\alpha_{R} i_{1} \alpha_{R}^{\prime} i_{1}^{\prime}}^{C_{2}-B_{1}}\right)_{\text {new }}=\sum_{i_{R} i_{R}^{\prime} i_{2} i_{2}{ }^{\prime}} v_{i_{R} i_{2}}^{\alpha_{R}}\left(v_{i_{R}^{\prime} i_{2}}^{\alpha_{R}{ }^{\prime}}\right)^{*}\left\{H_{i_{1} i_{2} i_{1}^{\prime} i_{2}^{\prime}}^{C_{1}-C_{2}} \delta_{i_{R} i_{R}^{\prime}}+H_{i_{R} i_{1} i_{R}^{\prime} i_{1}^{\prime}}^{C_{1}-B_{R}} \delta_{i_{2} i_{2}^{\prime}}\right\} .
\end{aligned}
$$

The long range interactions $\left(H^{B_{L}-C_{2}}\right)_{\text {new }},\left(H^{C_{1}-B_{R}}\right)_{\text {new }}$ and $\left(H^{B_{L}-B_{R}}\right)_{\text {new }}$ are not simply obtained by transforming the previous Hamiltonian, because they are defined after we add new single sites between the two blocks. These terms are constructed from the operators represented by the basis states of the new blocks and the single sites added in the system. The new Hamiltonian of six sites is then written by

$$
\begin{aligned}
(H)_{\text {new }}= & \left(H^{B_{L}}\right)_{\text {new }}+H^{C_{1}}+H^{C_{2}}+\left(H^{B_{R}}\right)_{\text {new }} \\
& +\left(H^{L-C 1}\right)_{\text {new }}+H^{C_{1}-C_{2}}+\left(H^{C_{2}-B_{R}}\right)_{\text {new }} \\
& +\left(H^{B_{L}-C_{2}}\right)_{\text {new }}+\left(H^{C_{1}-B_{R}}\right)_{\text {new }}+\left(H^{B_{L}-B_{R}}\right)_{\text {new }} .
\end{aligned}
$$

This Hamiltonian has similar structure to the previous one written in equation (10), and we repeat the above procedure until we obtain the desired size of system. Since there is no limitation on the size of system, this algorithm is called the infinite system algorithm of the DMRG. 


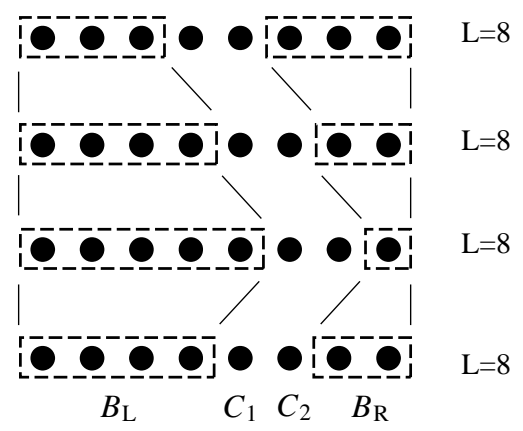

Figure 4. Schematic diagram for finite system algorithm of the DMRG.

\subsection{Finite system algorithm}

In the infinite system algorithm of the DMRG we calculate the Hamiltonian of any size of systems by iteratively extending the blocks. However, in order to deal with exponential increase in the number the basis states, we truncate the basis states. This truncation introduces an artificial error in the wavefunction, and we need to minimize the error by reconstructing the basis states within a fixed size of system. The algorithm to find the optimal basis states in a finite system is called the finite system algorithm of the DMRG. Here, we describe how we improve the ground-state wavefunction using the finite system algorithm of the DMRG.

Suppose we have extended the system up to $2 N+2$ sites by using infinite system algorithm of the DMRG. The left and right blocks now contain $N$ sites and the system is written by

$$
\mathrm{B}_{L}(N) \bullet \bullet \mathrm{B}_{R}(N) .
$$

Here $B_{L}(N)$ and $B_{R}(N)$ represent the left and the right blocks which contain $N$ sites and $\bullet$ represents a single site between the two blocks. In order to reconstruct the right block first, we extend only the left block and reduce the right block, as shown in figure 4. We calculate the optimal basis states for $B_{L}(N+1)$ by diagonalizing the density matrix calculated from the ground-state wavefunction of the system $B_{L}(N) \bullet \bullet B_{R}(N)$, and we obtain the Hamiltonian $H^{B_{L}}$ for the left block $B_{L}(N+1)$ by a similar calculation to that in the infinite system algorithm of the DMRG. We do not need to calculate the basis states and the Hamiltonian $H^{B_{R}}$ for $B_{R}(N-1)$ because they have already been obtained in the infinite system algorithm of the DMRG. The system of $2 N+2$ sites is now represented by

$$
\mathrm{B}_{L}(N+1) \bullet \bullet \mathrm{B}_{R}(N-1) .
$$

We repeat the extension of the left block until the right block becomes single site. Then, we extend the right block from the single site to reconstruct the basis states in $B_{R}$. Now the basis states of the right block are always determined from the groundstate wavefunction of $2 N+2$ sites. This is different from the calculation in the infinite system algorithm, where the size of system increases with the extension of the blocks. 
This difference improves the ground-state wavefunction obtained in the infinite system algorithm of the DMRG.

The extension of the right block is continued until the left block is reduced down to the single site. We then extend the left block to reconstruct the basis states in $B_{L}$. We repeat such sweeps until the ground-state energy and its wavefunction converge. Since we have reduced Hilbert space of the Hamiltonian by restricting the basis states, the obtained ground-state energy is always higher than the exact one. The increase in the number of the basis states systematically lowers the ground-state energy and its value approaches to the exact one. Thus the finite system algorithm of the DMRG method is equivalent to a variational method in which a trial wavefunction is constructed automatically under the restriction on the number of basis states.

\subsection{Evaluation of physical quantities}

We evaluate various physical quantities from the wavefunction obtained by the DMRG calculations. Since the basis states of the wavefunction alter after the reconstructions of the blocks, we need the operators represented in the basis states of the wavefunction. These operators are obtained by successive transformations of basis states starting from the operators defined in the initial single site. At each reconstruction of the blocks the operators such as $S_{i}^{z}$ is transformed as follows. If the $i$ th site is the single site next to the block $B_{L},\left(S_{i}^{z}\right)_{i_{1} i_{1}}$ is transformed by

$$
\left(S_{i}^{z}\right)_{\alpha_{L} \alpha_{L}{ }^{\prime}}=\sum_{i_{L} i_{1} i_{1}{ }^{\prime}} v_{i_{L} i_{1}}^{\alpha_{L}}\left(v_{i_{L} i_{1}}^{\alpha_{L}{ }^{\prime}}\right)^{*}\left(S_{i}^{z}\right)_{i_{1} i_{1}{ }^{\prime}}
$$

where extended new left block include the $i$ th site and has basis states $\left|\alpha_{L}\right\rangle$, and $v_{i_{L} i_{1}}^{\alpha_{L}}$ is the eigenvectors of the density matrix defined in equation (14). After the $i$ th site is included in the block $B_{L}, S_{i}^{z}$ is transformed by

$$
\left(S_{i}^{z}\right)_{\alpha_{L} \alpha_{L}{ }^{\prime}}=\sum_{i_{L} i_{L^{\prime} i_{1}}} v_{i_{L} i_{1}}^{\alpha_{L}}\left(v_{i_{L}^{\prime} i_{1}}^{\alpha_{L}^{\prime}}\right)^{*}\left(S_{i}^{z}\right)_{i_{L} i_{L}{ }^{\prime}} .
$$

The product of the two operators at sites $i$ and $j$, such as $S_{i}^{z} S_{j}^{z}$ are transformed by

$$
\left(S_{i}^{z} S_{j}^{z}\right)_{\alpha_{L} \alpha_{L^{\prime}}}=\sum_{i_{L} i_{L^{\prime}} i_{1} i_{1}{ }^{\prime}} v_{i_{L} i_{1}}^{\alpha_{L}}\left(v_{i_{L^{\prime}} i_{1}{ }^{\prime}}^{\alpha^{\prime}}\right)^{*}\left(S_{i}^{z}\right)_{i_{L} i_{L^{\prime}}}\left(S_{j}^{z}\right)_{i_{1} i_{1}{ }^{\prime}}
$$

where the $j$ th site is the single site next to the original left block $B_{L}$ and the $i$ th site is in the left block $B_{L}$. If both the $i$ th and $j$ th sites are in the left block $B_{L}$, they are transformed by

$$
\left(S_{i}^{z} S_{j}^{z}\right)_{\alpha_{L} \alpha_{L}{ }^{\prime}}=\sum_{i_{L} i_{L^{\prime} i_{1}}} v_{i_{L} i_{1}}^{\alpha_{L}}\left(v_{i_{L^{\prime}} i_{1}}^{\alpha_{L_{1}}}\right)^{*}\left(S_{i}^{z} S_{j}^{z}\right)_{i_{L} i_{L^{\prime}}}
$$

After we have obtained the operators represented by the basis states of the wavefunction $\Psi_{i_{L} i_{1} i_{2} i_{R}}\left|i_{L}\right\rangle\left|i_{1}\right\rangle\left|i_{2}\right\rangle\left|i_{R}\right\rangle$, we evaluate physical quantities. The local quantities $\left\langle\Psi\left|S_{i}^{z}\right| \Psi\right\rangle$ and correlation functions $\left\langle\Psi\left|S_{i}^{z} S_{j}^{z}\right| \Psi\right\rangle$ are given by

$$
\begin{aligned}
& \left\langle\Psi\left|S_{i}^{z}\right| \Psi\right\rangle=\sum_{i_{L} i_{L}^{\prime} i_{1} i_{2} i_{R}} \Psi_{i_{L}^{\prime} i_{1} i_{2} i_{R}}^{*}\left(S_{i}^{z}\right)_{i_{L} i_{L}} \Psi_{i_{L} i_{1} i_{2} i_{R}} \\
& \left\langle\Psi\left|S_{i}^{z} S_{j}^{z}\right| \Psi\right\rangle=\sum_{i_{L} i_{L^{\prime}} i_{1} i_{2} i_{R}} \Psi_{i_{L}^{\prime} i_{1} i_{2} i_{R}}^{*}\left(S_{i}^{z} S_{j}^{z}\right)_{i_{L} i_{L}} \Psi_{i_{L} i_{1} i_{2} i_{R}}
\end{aligned}
$$




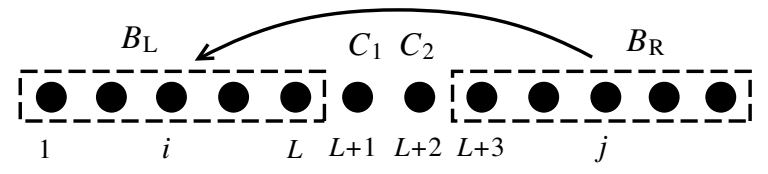

Figure 5. Long range hopping between the two blocks.

where both $S_{i}$ and $S_{j}$ are assumed to be in the left block. If $S_{j}$ is in the right block while $S_{i}$ is in the left block, the correlation function $\left\langle\Psi\left|S_{i}^{z} S_{j}^{z}\right| \Psi\right\rangle$ is given by

$$
\left\langle\Psi\left|S_{i}^{z} S_{j}^{z}\right| \Psi\right\rangle=\sum_{i_{L} i_{L^{\prime}}^{\prime} i_{1} i_{2} i_{R} i_{R^{\prime}}} \Psi_{i_{L}^{\prime} i_{1} i_{2} i_{R^{\prime}}}^{*}\left(S_{i}^{z}\right)_{i_{L} i_{L}}\left(S_{j}^{z}\right)_{i_{R}{ }^{\prime} i_{R}} \Psi_{i_{L} i_{1} i_{2} i_{R}} .
$$

\subsection{Fermion sign}

When an electron hops from one site to another, the fermion sign appears in the matrix element between the two basis states. The fermion sign depends on the number of electrons between the two sites, and we need to count the electrons. However, the number of electrons at each site in the basis states of the blocks is neither one nor zero after we have transformed the basis states using the eigenvectors of the density matrix. In order to correctly deal with the fermion sign, it is useful to define new creation (annihilation) operators, $\bar{c}_{i \sigma}^{\dagger}$ and $\left(\bar{c}_{i \sigma}\right)$, which include the fermion sign generated by the hopping from the $i$ th site to one end of the block. If we align the up-spin electrons and down-spin electrons separately, $\bar{c}_{i \sigma}^{\dagger}$ in the left block are defined by

$$
\bar{c}_{i \sigma}^{\dagger} \equiv c_{i \sigma}^{\dagger}(-1)^{N_{i+1 \sigma}+N_{i+2 \sigma}+\cdots+N_{L \sigma}}
$$

where $L$ is the number of sites in the left block (see figure 51) and $N_{i \sigma}$ are the number operators of the electron with spin $\sigma$ at the $i$ th site. The operators $\bar{c}_{j \sigma}^{\dagger}$ in the right block are defined by

$$
\bar{c}_{j \sigma}^{\dagger} \equiv c_{j \sigma}^{\dagger}(-1)^{N_{L+3 \sigma}+N_{L+4 \sigma}+\cdots+N_{j-1 \sigma}} .
$$

The matrix element $\left\langle k\left|c_{i \sigma}^{\dagger} c_{j \sigma}\right| l\right\rangle$ generated by a long-range hopping between the two blocks is then given by

$$
\left\langle k\left|c_{i \sigma}^{\dagger} c_{j \sigma}\right| l\right\rangle=\left(\bar{c}_{i \sigma}^{\dagger}\right)_{k_{L} l_{L}}\left(\bar{c}_{j \sigma}\right)_{k_{R} l_{R}}(-1)^{\left(N_{L+1 \sigma}\right)_{1}+\left(N_{L+2 \sigma}\right)_{l_{2}}} \delta_{k_{1} l_{1}} \delta_{k_{2} l_{2}}
$$

where $|l\rangle \equiv\left|l_{L}\right\rangle\left|l_{1}\right\rangle\left|l_{2}\right\rangle\left|l_{R}\right\rangle$. The coefficient $(-1)^{\left(N_{L+1 \sigma}\right)_{1}+\left(N_{L+2 \sigma}\right) l_{2}}$ is the fermion sign coming from the electrons with spin $\sigma$ in the two single sites between the blocks. In this expression we need not calculate the fermion sign coming from the electrons in the blocks, and the matrix elements of long-range hoppings between the two blocks are obtained by only counting electrons in the two single sites between the two blocks.

The operators $\bar{c}_{i \sigma}^{\dagger}$ are obtained by successive transformations starting from the original operators defined in the single site. When the left block is enlarged by including the $i_{1}$ th site, $\bar{c}_{i \sigma}^{\dagger}$ in the left block is transformed as

$$
\left(\bar{c}_{i \sigma}^{\dagger}\right)_{\alpha_{L} \alpha_{L}}=\sum_{i_{L} i_{L}^{\prime} i_{1}} v_{i_{L} i_{1}}^{\alpha_{L}}\left(v_{i_{L}^{\prime} i_{1}}^{\alpha_{L^{\prime}}{ }^{\prime}}\right)^{*}\left(\bar{c}_{i \sigma}^{\dagger}\right)_{i_{L} i_{L^{\prime}}}(-1)^{N_{i_{1} \sigma}}
$$


where $(-1)^{N_{i_{1} \sigma}}$ is the fermion sign generated by the electron at the $i_{1}$ th site, and $\bar{c}_{i \sigma}^{\dagger} \equiv c_{i \sigma}^{\dagger}$ when the $i$ th site is the right end of the left block, $i=L$. Since the basis states $\left|l_{1}\right\rangle$ of the single site are the eigenstates of the number operator $N_{i_{1}}, N_{i_{1}}$ is either one or zero depending on the index $l_{1}$.

\subsection{Conserved quantities}

In the DMRG calculation, most of the computing time is consumed for the diagonalization of the Hamiltonian. Thus, it is important to use an efficient method for the diagonalization. Here we consider the basis states classified by the conserved quantities of the Hamiltonian. By using these basis states we need only diagonalize the Hamiltonian within a subspace specified by a set of quantum numbers. For example, Heisenberg model without transverse magnetic field conserves the $z$-component of the total spin, and all the eigenstates of the Hamiltonian are classified by total $S^{z}$. The blocks $B_{L}(2)$ and $B_{R}(2)$ containing two $S=1 / 2$ spins have basis states $|\uparrow, \uparrow>,| \uparrow, \downarrow>$, $\mid \downarrow, \uparrow>$, and $\mid \downarrow, \downarrow>$ with quantum numbers of $S^{z}=1,0,0$, and -1 , respectively, and the basis states of the total system $B^{L}(2) \bullet \bullet B^{R}(2)$ are constructed from various combinations of local basis states which satisfy

$$
S_{\text {tot }}^{z}=S_{L}^{z}+S_{C 1}^{z}+S_{C 2}^{z}+S_{R}^{z}=\text { const. }
$$

When we calculate the ground state of $S_{\text {tot }}^{z}=0$, there are 10-configurations which are represented by $\left(S_{L}^{z}, S_{C 1}^{z}, S_{C 2}^{z}, S_{R}^{z}\right)=(1,1 / 2,-1 / 2,-1),(1,-1 / 2,1 / 2,-1),(1,-1 / 2,-1 / 2,0),(0,-$ $1 / 2,-1 / 2,1),(0,1 / 2,-1 / 2,0),(0,-1 / 2,1 / 2,0),(-1,1 / 2,1 / 2,0),(0,1 / 2,1 / 2,-1),(-1,1 / 2,-1 / 2,1)$, $(-1,-1 / 2,1 / 2,1)$. The number of total basis states is 20 , which is lower than $2^{6}=64$ of full Hilbert space. Since the Hamiltonian $H^{B_{L}}$ and $H^{B_{R}}$ conserve total $S^{z}$, they are also block diagonal, and $H^{B_{L}}+H^{C_{1}}+H^{C_{2}}+H^{B_{R}}$ have matrix elements only within each subspace specified by $\left(S_{B_{L}}^{z}, S_{C_{1}}^{z}, S_{C_{2}}^{z}, S_{B_{R}}^{z}\right)$. The matrix elements between different subspaces are generated only by the terms $H^{B_{-} C_{1}}+H^{C_{1}-C_{2}}+H^{C_{2}-B_{R}}+H^{B_{L}-C_{2}}+$ $H^{C_{1}-B_{R}}+H^{B_{L}-B_{R}}$.

The density matrix $\rho_{i i^{\prime}}=\sum_{j} \Psi_{i j}^{*} \Psi_{i^{\prime} j}$ obtained from the ground-state wavefunction is also block diagonal, because total $S^{z}$ of the basis states $j$ is uniquely determined by the basis states $i$ of the density matrix. This means that the density matrix does not have any matrix elements between different subspaces of total $S^{z}$. Since the density matrix is block diagonal, the unitary transformation of the basis states is defined only within the same subspace of total $S^{z}$, and the basis states of the new blocks are also classified by total $S^{z}$.

By using the basis states classified by the conserved quantities such as the zcomponent of the total spin and the number of electrons, the dimension of the Hamiltonian, operators, and density matrix are reduced. The reduction of the dimensions improves the efficiency and accuracy of the numerical calculation, and reduces the memory space needed in the calculation. 


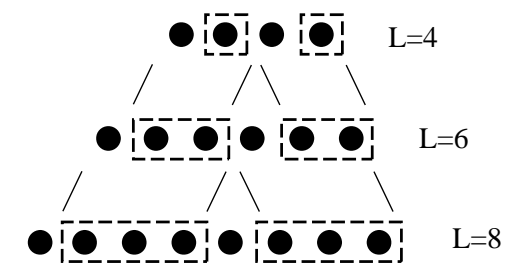

Figure 6. Arrangement of the blocks and the single sites for the periodic boundary conditions.

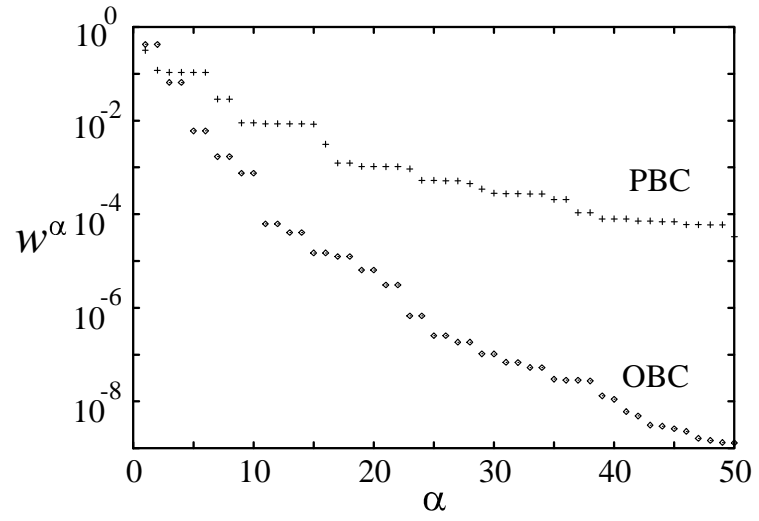

Figure 7. Eigenvalues of the density matrix for $\mathrm{S}=1 / 2$ Heisenberg model with 40 spins under the periodic boundary conditions $(\mathrm{PBC})$ and the open boundary conditions $(\mathrm{OBC})$.

\subsection{Periodic boundary conditions}

In the DMRG calculations, the open boundary conditions are usually used. However, the existence of the boundaries sometimes causes large boundary effects on the ground state. These boundary effects are removed when we impose the periodic boundary conditions. In this case, we usually align the blocks and single sites symmetrically as shown in figure 6. The truncation error under the periodic boundary conditions is generally large compared with that obtained in the open boundary conditions. To keep the accuracy of the results, we need much more basis states in each block. This is actually shown in figure [7, which shows slower decay of the eigenvalues of the density matrix for the periodic boundary conditions.

Under the periodic boundary conditions, the left and right blocks are connected with each other through both ends of the blocks. The reduction of the accuracy is caused by the fact that the interactions around the boundaries between the blocks and the single sites such as $H^{B_{L}-C_{1}}$ and $H^{C_{2}-B_{R}}$ are represented in the product space of the block and single site, and they are not renormalized to reproduce the ground-state wavefunction within a small number of basis states. When the interactions across the boundaries are weak, the accuracy of the DMRG calculation will be improved. 


\section{Finite temperature DMRG}

The zero-temperature DMRG method described in the above section enables us to calculate the ground state wavefunction of large-size of systems with controlled accuracy. However, in order to study the properties of the bulk system, we need to extend the system in real space direction and analyze the asymptotic behaviors. In the finitetemperature DMRG method, the thermodynamic quantities of an infinitely long system are directly obtained from the eigenvalues of QTM, and temperature dependence is obtained in the process of iterative extensions of the QTM in the $\beta$-direction. In this section we describe how we calculate thermodynamic quantities using the DMRG method applied to the QTM.

\subsection{Transfer matrix method}

The thermodynamic quantities are calculated from the partition function of the system. In one-dimensional quantum systems the partition function is obtained from the maximum eigenvalue of the QTM[46, 47]. Here we first briefly summarize the way to calculate the QTM and the partition function.

In the following we suppose that the Hamiltonian consists of only on-site and nearest-neighbor interactions so that we can divide the Hamiltonian into two parts $H_{\text {odd }}=\sum_{n=0}^{L / 2-1} h_{2 n+1,2 n+2}$ and $H_{\text {even }}=\sum_{n=0}^{L / 2-1} h_{2 n, 2 n+1}$. Here $h_{i, i+1}$ contains on-site and nearest-neighbor interactions between the $i$ th and $i+1$ th sites and each $h_{i, i+1}$ commutes with all other $h_{i+2 n, i+1+2 n}$ :

$$
\left[h_{2 n, 2 n+1}, h_{2 n^{\prime}, 2 n^{\prime}+1}\right]=\left[h_{2 n+1,2 n+2}, h_{2 n^{\prime}+1,2 n^{\prime}+2}\right]=0 .
$$

The partition function is then decomposed into a product of $e^{-\beta h_{i, i+1} / M}$ as

$$
\begin{aligned}
Z & =\operatorname{Tr} e^{-\beta H} \\
& =\lim _{M \rightarrow \infty} \operatorname{Tr}\left[e^{-\beta H_{\text {odd }} / M} e^{-\beta H_{\text {even }} / M}\right]^{M} \\
& =\lim _{M \rightarrow \infty} \operatorname{Tr}\left[\prod_{n=0}^{L / 2-1} e^{-\beta h_{2 n+1,2 n+2} / M} \prod_{n=0}^{L / 2-1} e^{-\beta h_{2 n, 2 n+1} / M}\right]^{M}
\end{aligned}
$$

where $M$ is the Trotter number [48, 49, 50].

Here we introduce imaginary time $\tau_{j}$, whose index $j$ takes only integers up to $2 M$, and we represent the partition function in a tensor product of $e^{-\beta h_{i, i+1} / M}$ under the complete basis set for $\tau_{j}$ as shown in figure $8(\mathrm{a})$, where $e^{-\beta h_{i, i+1} / M}$ is represented by a hatched square shown in figure 8 (b). Each $e^{-\beta h_{i, i+1} / M}$ is then represented by the basis states specified by $i, i+1$ and $\tau_{j}, \tau_{j+1}$. We explicitly write the imaginary time indices $\tau_{j}$ and $\tau_{j+1}$ in $e^{-\beta h_{i, i+1} / M}$, and define the tensor $e_{\left(\tau_{j+1}, \tau_{j}\right)}^{-\beta h_{i, i+1} / M}$ represented by

$$
\begin{aligned}
e_{\left(\tau_{j+1}, \tau_{j}\right)}^{-\beta h_{i, i+1} / M} \equiv & \sum_{\sigma_{i, \tau_{j+1}}} \sum_{\sigma_{i+1, \tau_{j+1}}} \sum_{\sigma_{i_{,}, \tau_{j}}^{\prime}} \sum_{\sigma_{i+1, \tau_{j}}^{\prime}} \\
& \left|\sigma_{i, \tau_{j+1}} \sigma_{i+1, \tau_{j+1}}\right\rangle\left\langle\sigma_{i} \sigma_{i+1}\left|e^{-\beta h_{i, i+1} / M}\right| \sigma_{i}^{\prime} \sigma_{i+1}^{\prime}\right\rangle\left\langle\sigma_{i, \tau_{j}}^{\prime} \sigma_{i+1, \tau_{j}}^{\prime}\right|
\end{aligned}
$$


(a)

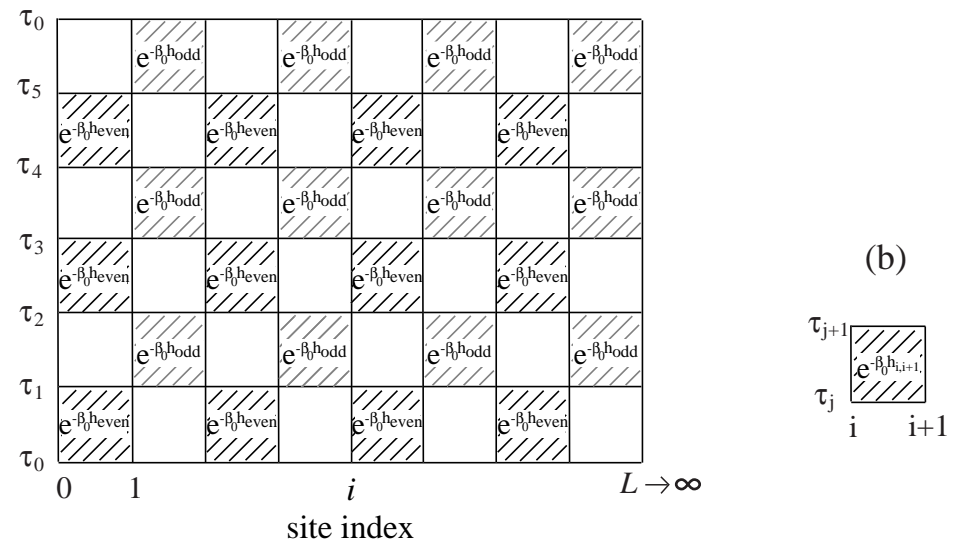

Figure 8. (a) Partition function $Z$ for $M=3$ represented by a tensor product of $e^{-\beta_{0} h_{\text {even }}}$ and $e^{-\beta_{0} h_{\text {odd }}}$, where $h_{\text {even }}=h_{2 n, 2 n+1}$ and $h_{\text {odd }}=h_{2 n+1,2 n+2}$. (b) Graphical representation of $e_{\left(\tau_{j+1}, \tau_{j}\right)}^{-\beta_{0} h_{i, i+1}}$ with $\beta_{0}=\beta / M$.

where $\sigma_{i, \tau_{j}}$ is the local variable which specifies the state at the $i$ th site and at imaginary time $\tau_{j}$.

The QTM $\mathcal{T}_{n}$ is written by

$$
\mathcal{T}_{n}=\lim _{M \rightarrow \infty} \prod_{j=0}^{M-1}\left(e_{\left(\tau_{2 j+2}, \tau_{2 j+1}\right)}^{-\beta h_{2 n+1,2 n+2} / M} e_{\left(\tau_{2 j+1}, \tau_{2 j}\right)}^{-\beta h_{2 n, 2 n+1} / M}\right)
$$

as a tensor product of $e_{\left(\tau_{j+1}, \tau_{j}\right)}^{-\beta h_{i, i+1} / M}$ with the periodic boundary conditions in the $\beta$ direction, $\tau_{2 M}=\tau_{0}$. This matrix $\mathcal{T}_{n}$ is graphically represented in figure 9. When the system is translationally symmetric, we can omit the site index $n$ in $\mathcal{T}_{n}$, and the partition function is given by

$$
Z=\operatorname{Tr} \mathcal{T}^{L / 2}
$$

Using the eigenvectors of the transfer matrix as basis states, we can represent the partition function using their eigenvalues $\lambda_{i}$ as

$$
Z=\sum_{i} \lambda_{i}^{L / 2}
$$

In the thermodynamic limit $L \rightarrow \infty, Z$ is determined only by the maximum eigenvalue $\lambda_{\max }$ :

$$
Z=\lambda_{\max }^{L / 2}
$$

The free energy par site is then calculated from the maximum eigenvalue $\lambda_{\max }$ as

$$
\begin{aligned}
F & =-\ln Z /(L \beta) \\
& =-\ln \lambda_{\max } /(2 \beta) .
\end{aligned}
$$

The thermodynamic quantities such as the entropy, specific heat and the magnetic susceptibility are given by taking the derivative of $F$ with respect to the temperature and magnetic field. 


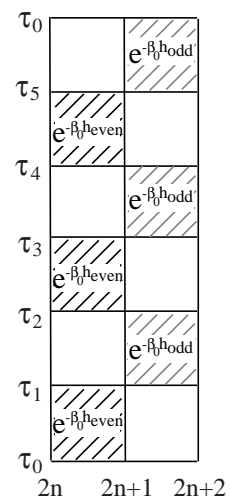

Figure 9. Quantum transfer matrix $\mathcal{T}$ represented by a tensor product of $e^{-\beta h_{i, i+1} / M}$ with $M=3$.

\subsection{Application of the DMRG to the quantum transfer matrix}

In order to calculate low temperature properties, we need to increase the Trotter number $M$ keeping $\beta / M=\Delta \tau$ sufficiently small. However, the dimension of the QTM exponentially increases with increasing the Trotter number. In this section we describe how we apply the DMRG method to QTM and obtain the maximum eigenvalue for large $M$.

In the zero-temperature DMRG method, we extend the Hamiltonian in the real space direction by restricting the number of basis states by using the eigenvectors of the density matrix calculated from the ground-state wavefunction. In the finite-temperature DMRG method, we extend the transfer matrix in the $\beta$-direction by restricting the basis states using the density matrix calculated from the eigenvector of the maximum eigenvalue $\lambda_{\max }$.

We start from a small QTM with $M=2$ and divide it into two blocks, $\mathcal{T}^{A(M=1)}$ and $\mathcal{T}^{B(M=1)}$, as

$$
\begin{aligned}
\mathcal{T}_{\left(\tau_{2}, \tau_{1}, \tau_{0}\right)}^{A(M=1)} & =\mathcal{T}_{\left(\tau_{2}, \tau_{1}, \tau_{0}\right)}^{B(M=1)} \\
& =e_{\left(\tau_{2}, \tau_{1}\right)}^{-\beta_{0} h_{\text {odd }}} e_{\left(\tau_{1}, \tau_{0}\right)}^{-\beta_{0} h_{\text {even }}}
\end{aligned}
$$

where $h_{\text {odd }}=h_{2 n+1,2 n+2}$ and $h_{\text {even }}=h_{2 n, 2 n+1}$. The transfer matrix $\mathcal{T}$ is defined by

$$
\begin{array}{ll}
\mathcal{T}^{(2 M)}=\mathcal{T}_{\left(\tau_{0}, \tau_{(4 M-1, \cdots, 2 M+2,2 M+1)}, \tau_{2 M}\right)}^{B(M)} & \mathcal{T}_{\left(\tau_{2 M}, \tau_{(2 M-1, \cdots, 2,1)}, \tau_{0}\right)}^{A(M)} \\
\mathcal{T}^{(2 M+1)}=\mathcal{T}_{\left(\tau_{0}, \tau_{(4 M+1, \cdots, 2 M+3,2 M+2)}, \tau_{2 M+1}\right)}^{B(M+1 / 2)} & \mathcal{T}_{\left(\tau_{2 M+1}, \tau_{(2 M, \cdots, 2,1)}, \tau_{0}\right)}^{A(M+1 / 2)}
\end{array}
$$

depending on the parity of total Trotter number, and we have imposed the periodic boundary conditions, $\sigma_{i, \tau_{4 M}}=\sigma_{i, \tau_{0}}$, in the $\beta$-direction. We extend $\mathcal{T}^{A}$ and $\mathcal{T}^{B}$ as

$$
\begin{array}{ll}
e_{\left(\tau_{2 M+1}, \tau_{2 M}\right)}^{-\beta_{0} h_{\text {even }}} \mathcal{T}_{\left(\tau_{2 M}, \tau_{(2 M-1, \cdots, 2,1)}, \tau_{0}\right)}^{A(M)} & \rightarrow \mathcal{T}_{\left(\tau_{2 M+1}, \tau_{(2 M, \cdots, 2,1)}, \tau_{0}\right)}^{A(M+1 / 2)} \\
\mathcal{T}_{\left(\tau_{2 M+2}, \tau_{(2 M+1, \cdots, 4,3)}, \tau_{2}\right)}^{B(M)} e_{\left(\tau_{2}, \tau_{1}\right)}^{-\beta_{0} h_{\text {odd }}} & \rightarrow \mathcal{T}_{\left(\tau_{2 M+2}, \tau_{(2 M+1, \cdots, 3,2)}, \tau_{1}\right)}^{B(M+1 / 2)} \\
e_{\left(\tau_{2 M+2}, \tau_{2 M+1}\right)}^{-\beta_{0} h_{\text {odd }}} \mathcal{T}_{\left(\tau_{2 M+1}, \tau_{(2 M, \cdots, 2,1)}, \tau_{0}\right)}^{A(M+1 / 2)} & \rightarrow \mathcal{T}_{\left(\tau_{2 M+2}, \tau_{(2 M+1, \cdots, 2,1)}, \tau_{0}\right)}^{A(M+1)} \\
\mathcal{T}_{\left(\tau_{2 M+2}, \tau_{(2 M+2, \cdots, 3,2)}, \tau_{1}\right)}^{B(M+1 / 2)} e_{\left(\tau_{1}, \tau_{0}\right)}^{-\beta_{0} \operatorname{loven}_{2}} & \rightarrow \mathcal{T}_{\left(\tau_{2 M+2}, \tau_{(2 M+1, \cdots, 2,1)}, \tau_{0}\right)}^{B(M+1)}
\end{array}
$$



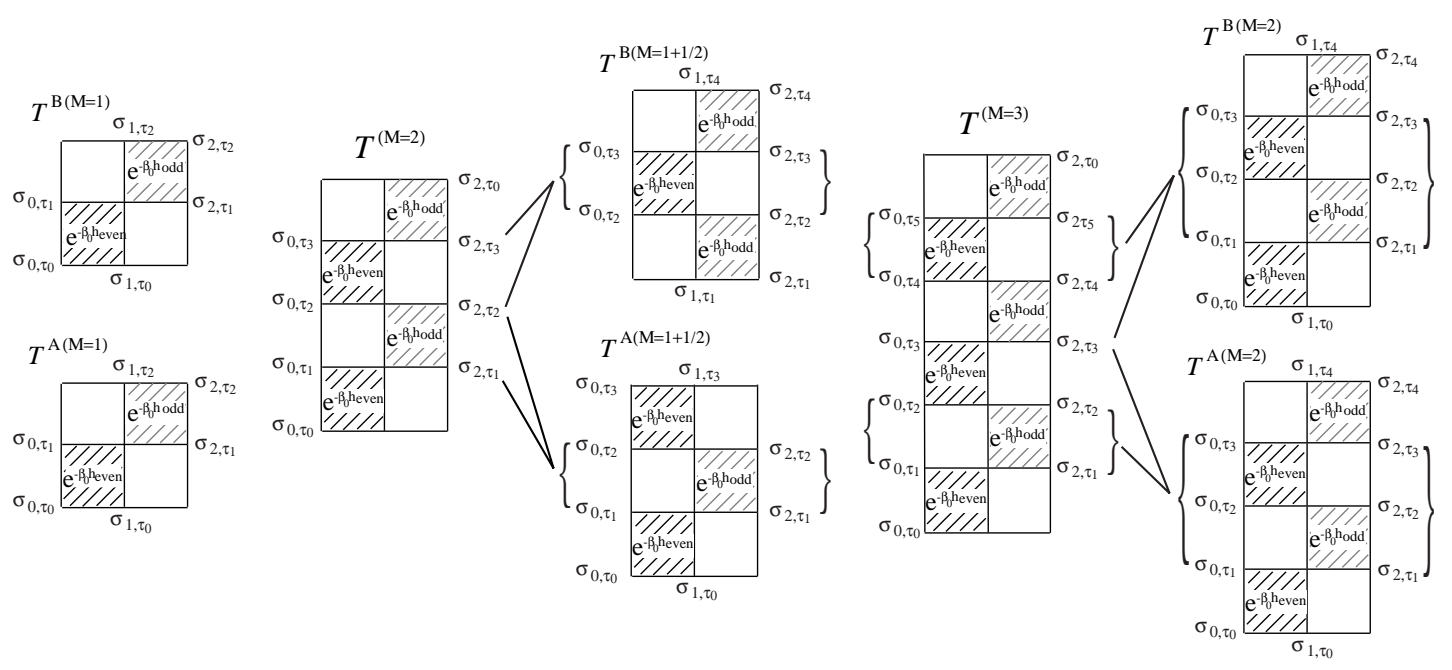

Figure 10. Schematic diagram for the expansion of the QTM.

as shown in figure 10 .

At each expansion of the QTM in the $\beta$-direction, we restrict the basis states of $\mathcal{T}^{A}$ and $\mathcal{T}^{B}$ while keeping the accuracy of $\lambda_{\max }$ and corresponding eigenvectors, which satisfy the following equations

$$
\begin{gathered}
\sum_{j} V_{j}^{L} \mathcal{T}_{j j^{\prime}}=\lambda_{\max } V_{j^{\prime}}^{L} \\
\sum_{j^{\prime}} \mathcal{T}_{j j^{\prime}} V_{j^{\prime}}^{R}=\lambda_{\max } V_{j}^{R}
\end{gathered}
$$

where $\mathcal{T}_{j j^{\prime}}$ represents the matrix element of $\mathcal{T}$ between the basis states $j$ and $j^{\prime}$, and $V^{L}$ and $V^{R}$ are the left and the right eigenvectors of the maximum eigenvalue $\lambda_{\max }$, normalized by $\left\langle V^{L} \mid V^{R}\right\rangle \equiv 1 . \quad V^{L}$ and $V^{R}$ are usually different because $\mathcal{T}$ is nonHermitian.

Since the maximum eigenvalue $\lambda_{\max }$ of $\mathcal{T}$ is equivalent to $\left\langle V^{L}|\mathcal{T}| V^{R}\right\rangle$, we need to keep the norm $\left\langle V^{L} \mid V^{R}\right\rangle$ as much as possible when we restrict the basis states. To find the optimal basis states of $\mathcal{T}^{A}$, we calculate the following density matrix defined by

$$
\rho_{k k^{\prime}}^{A}=\sum_{l} V_{k l}^{L} V_{k^{\prime} l}^{R}
$$

where index $k$ represents the basis states correspond to the imaginary time between $\tau_{1}$ and $\tau_{2 M}$ (between $\tau_{1}$ and $\tau_{2 M-1}$ ) for $\mathcal{T}^{A(M+1 / 2)}\left(\mathcal{T}^{A(M)}\right)$, which is transformed to new basis states, and $l$ represents the basis states for the remaining imaginary time: $\{j\}=\{k, l\}$. Since $\left\langle V^{L} \mid V^{R}\right\rangle$ is given by the sum of the eigenvalues $w^{\alpha}$ of the density matrix, $\left\langle V^{L} \mid V^{R}\right\rangle=\operatorname{Tr} \rho^{A}=\sum_{k} \rho_{k k}^{A}=\sum_{\alpha} w_{\alpha}$, the optimal basis set within a fixed number $m$ of basis states are obtained from the eigenvectors of the $m$ largest eigenvalues. The new basis states are thus defined by the eigenvectors $\vec{v}^{\alpha(L)}$ and $\vec{v}^{\alpha(R)}$ of the density matrix, which satisfy the eigenvalue equations

$$
\sum_{k} v_{k}^{\alpha(L)} \rho_{k k^{\prime}}^{A}=w^{\alpha} v_{k^{\prime}}^{\alpha(L)}
$$




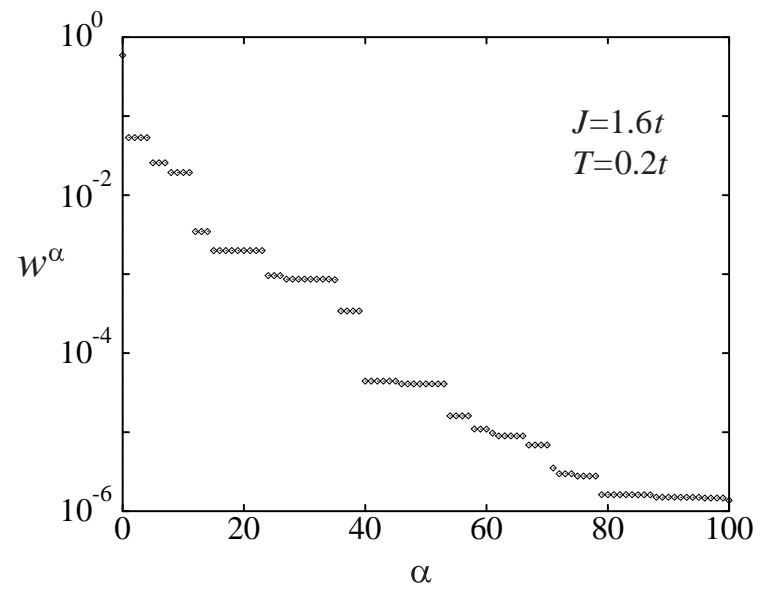

Figure 11. Eigenvalues of the density matrix $\rho$ calculated from the eigenvectors for $\lambda_{\max }$ of the QTM in one-dimensional Kondo lattice model at half-filling.

$$
\sum_{k^{\prime}} \rho_{k k^{\prime}}^{A} v_{k^{\prime}}^{\alpha(R)}=w^{\alpha} v_{k}^{\alpha(R)}
$$

By using $\vec{v}^{\alpha(L)}$ and $\vec{v}^{\alpha(R)}$ we transform $\mathcal{T}^{A}$ as

$$
\mathcal{T}_{\alpha \alpha^{\prime}}^{A}=\sum_{k k^{\prime}} v_{k}^{\alpha(R)} \mathcal{T}_{k k^{\prime}}^{A} v_{k^{\prime}}^{\alpha^{\prime}(L)}
$$

Here, we have omitted the indices of the basis states corresponding to the part of the Hilbert space with imaginary time $\tau_{0}$ and $\tau_{2 M+1}\left(\tau_{0}\right.$ and $\left.\tau_{2 M}\right)$ for $\mathcal{T}^{A(M+1 / 2)}\left(\mathcal{T}^{A(M)}\right)$, which are included in $\mathcal{T}^{A}$ but independent of the transformation. As a result of the non-Hermitian density matrix, the left and the right eigenvectors, $\vec{v}^{\alpha(L)}$ and $\vec{v}^{\alpha(R)}$, are different, and this difference makes a different transformation between the left and right basis states of the transfer matrix. Since the left and right eigenvectors $\vec{v}^{\alpha(L)}$ and $\vec{v}^{\alpha(R)}$ are dual orthogonal

$$
\sum_{k} v_{k}^{\alpha(L)} v_{k}^{\alpha^{\prime}(R)}=\delta_{\alpha \alpha^{\prime}}
$$

the orthogonality of the left and right basis states is retained.

In this way, we restrict the number of basis states while keeping the accuracy of the maximum eigenvalue and its eigenvectors. The truncation error in the calculation is estimated from the sum of the eigenvalues of the density matrix which are truncated off, and it is given by $1-\sum_{k=1}^{m} w^{k}$, where $w^{k}$ is the $k$ th largest eigenvalue. In figure 11] we show a typical example of the eigenvalues of the density matrix. We can see only a small number of eigenstates have large eigenvalues and the truncation error is about $10^{-4}$ for $m=50$. In such a case, we can repeat the expansion of the QTM and obtain its maximum eigenvalue with controlled accuracy.

\subsection{Quantum transfer matrix}

Here we explicitly write the QTM. The QTM is obtained by extracting the matrix related to the site indices $2 n, 2 n+1$, and $2 n+2$ from the partition function decomposed 
into a tensor product of $e_{\left(\tau_{2 m+2}, \tau_{2 m+1}\right)}^{-\beta_{0} h_{2 n+1,2 n+2}}$ and $e_{\left(\tau_{2 m+1}, \tau_{2 m}\right)}^{-\beta_{0} h_{2 n+2 n+1}}$. We describe these local tensors using the local variables $\sigma_{i, \tau_{j}}$ at site $i$ and imaginary time $\tau_{j} . \sigma$ represents up-spin state or down-spin state for $S=1 / 2$ Heisenberg model. The transfer matrix $\mathcal{T}^{(M)}$ is then written by

$$
\begin{aligned}
& \mathcal{T}^{(M)}=\sum_{\sigma_{1, \tau_{0}} \cdots \sigma_{1, \tau_{2 M-1}}} \Theta\left(\sigma_{1, \tau_{0}} \sigma_{2, \tau_{0}} \mid \sigma_{1, \tau_{2 M-1}} \sigma_{2, \tau_{2 M-1}}\right) \cdots \\
& \Theta\left(\sigma_{0, \tau_{3}} \sigma_{1, \tau_{3}} \mid \sigma_{0, \tau_{2}} \sigma_{1, \tau_{2}}\right) \Theta\left(\sigma_{1, \tau_{2}} \sigma_{2, \tau_{2}} \mid \sigma_{1, \tau_{1}} \sigma_{2, \tau_{1}}\right) \Theta\left(\sigma_{0, \tau_{1}} \sigma_{1, \tau_{1}} \mid \sigma_{0, \tau_{0}} \sigma_{1, \tau_{0}}\right)
\end{aligned}
$$

where

$$
\Theta\left(\sigma_{i, \tau_{j+1}} \sigma_{i+1, \tau_{j+1}} \mid \sigma_{i, \tau_{j}} \sigma_{i+1, \tau_{j}}\right)=\left\langle\sigma_{i, \tau_{j+1}}, \sigma_{i+1, \tau_{j+1}}\left|e_{\left(\tau_{j+1}, \tau_{j}\right)}^{-\beta_{0} h_{\mathrm{i}+1}}\right| \sigma_{i, \tau_{j}}, \sigma_{i+1, \tau_{j}}\right\rangle
$$

and $\beta_{0}=\beta / M=2\left(\tau_{j+1}-\tau_{j}\right)=\Delta \tau$.

The matrix elements $\Theta\left(\sigma_{i, \tau_{j+1}} \sigma_{i+1, \tau_{j+1}} \mid \sigma_{i, \tau_{j}} \sigma_{i+1, \tau_{j}}\right)$ are determined by using eigenstates of the local Hamiltonian $h_{i, i+1}$. In the case of $S=1 / 2$ Heisenberg model, $h_{i, i+1}$ is $J \mathbf{S}_{i} \cdot \mathbf{S}_{i+1}$. The eigenvalues $E_{m}$ are $(-3 J / 4, J / 4, J / 4, J / 4)$ and the corresponding eigenvectors are

$$
\begin{aligned}
& \left|V_{1}\right\rangle=\frac{1}{\sqrt{2}}(|\uparrow, \downarrow\rangle-|\downarrow, \uparrow\rangle) \\
& \left|V_{2}\right\rangle=\frac{1}{\sqrt{2}}(|\uparrow, \downarrow\rangle+|\downarrow, \uparrow\rangle) \\
& \left|V_{3}\right\rangle=|\uparrow, \uparrow\rangle \\
& \left|V_{4}\right\rangle=|\downarrow, \downarrow\rangle .
\end{aligned}
$$

The matrix element $\left\langle\uparrow_{i}, \downarrow_{i+1}\left|e^{-\beta_{0} h_{\mathrm{i}, \mathrm{i}+1}}\right| \uparrow_{i}, \downarrow_{i+1}\right\rangle$ is then given by

$$
\begin{aligned}
& \left\langle\uparrow_{i}, \downarrow_{i+1}\left|e^{-\beta_{0} h_{\mathrm{i}, \mathrm{i}+1}}\right| \uparrow_{i}, \downarrow_{i+1}\right\rangle \\
& =\sum_{m}\left\langle\uparrow_{i}, \downarrow_{i+1} \mid V_{m}\right\rangle\left\langle V_{m}\left|e^{-\beta_{0} E_{m}}\right| V_{m}\right\rangle\left\langle V_{m} \mid \uparrow_{i}, \downarrow_{i+1}\right\rangle \\
& =\frac{1}{2}\left(e^{\beta_{0} 3 J / 4}+e^{-\beta_{0} J / 4}\right) .
\end{aligned}
$$

Similarly, off-diagonal elements and other diagonal elements are obtained as follows:

$$
\begin{aligned}
\left\langle\uparrow_{i}, \downarrow_{i+1}\left|e^{-\beta_{0} h_{\mathrm{i}, \mathrm{i}+1}}\right| \downarrow_{i}, \uparrow_{i+1}\right\rangle & =\left\langle\downarrow_{i}, \uparrow_{i+1}\left|e^{-\beta_{0} h_{\mathrm{i}, \mathrm{i}+1}}\right| \uparrow_{i}, \downarrow_{i+1}\right\rangle \\
& =-\frac{1}{2}\left(e^{\beta_{0} 3 J / 4}-e^{-\beta_{0} J / 4}\right) \\
\left\langle\uparrow_{i}, \uparrow_{i+1}\left|e^{-\beta_{0} h_{\mathrm{i}, \mathrm{i}+1}}\right| \uparrow_{i}, \uparrow_{i+1}\right\rangle & =\left\langle\downarrow_{i}, \downarrow_{i+1}\left|e^{-\beta_{0} h_{\mathrm{i}, \mathrm{i}+1}}\right| \downarrow_{i}, \downarrow_{i+1}\right\rangle \\
& =e^{-\beta_{0} J / 4} .
\end{aligned}
$$

Thus, the matrix $\Theta\left(\sigma_{i, \tau_{j+1}} \sigma_{i+1, \tau_{j+1}} \mid \sigma_{i, \tau_{j}} \sigma_{i+1, \tau_{j}}\right)$ is represented by

$$
e^{\beta_{0} J / 4}\left(\begin{array}{cccc}
e^{-\beta_{0} J / 2} & 0 & 0 & 0 \\
0 & \cosh \left(-\beta_{0} J / 2\right) & \sinh \left(-\beta_{0} J / 2\right) & 0 \\
0 & \sinh \left(-\beta_{0} J / 2\right) & \cosh \left(-\beta_{0} J / 2\right) & 0 \\
0 & 0 & 0 & e^{-\beta_{0} J / 2}
\end{array}\right)
$$

with the basis states of the matrix

$$
\left(\left|\uparrow_{i, \tau_{j}}, \uparrow_{i+1, \tau_{j}}\right\rangle,\left|\uparrow_{i, \tau_{j}}, \downarrow_{i+1, \tau_{j}}\right\rangle,\left|\downarrow_{i, \tau_{j}}, \uparrow_{i+1, \tau_{j}}\right\rangle,\left|\downarrow_{i, \tau_{j}}, \downarrow_{i+1, \tau_{j}}\right\rangle\right) .
$$


In order to explicitly represent the QTM, we need to use the basis states

$$
\left(\left|\uparrow_{i, \tau_{j}}, \uparrow_{i, \tau_{j+1}}\right\rangle,\left|\uparrow_{i, \tau_{j}}, \downarrow_{i, \tau_{j+1}}\right\rangle,\left|\downarrow_{i, \tau_{j}}, \uparrow_{i, \tau_{j+1}}\right\rangle,\left|\downarrow_{i, \tau_{j}}, \downarrow_{i, \tau_{j+1}}\right\rangle\right)
$$

defined along the $\beta$-axis at a given site $i$. The matrix $\hat{\Theta}$ defined in these basis states is related to the matrix $\Theta$ as

$$
\hat{\Theta}\left(\sigma_{i, \tau_{j}} \sigma_{i, \tau_{j+1}} \mid \sigma_{i+1, \tau_{j}} \sigma_{i+1, \tau_{j+1}}\right)=\Theta\left(\sigma_{i, \tau_{j+1}} \sigma_{i+1, \tau_{j+1}} \mid \sigma_{i, \tau_{j}} \sigma_{i+1, \tau_{j}}\right)
$$

Thus, the matrix $\hat{\Theta}$ is represented by

$$
e^{\beta_{0} J / 4}\left(\begin{array}{cccc}
e^{-\beta_{0} J / 2} & 0 & 0 & \cosh \left(-\beta_{0} J / 2\right) \\
0 & 0 & \sinh \left(-\beta_{0} J / 2\right) & 0 \\
0 & \sinh \left(-\beta_{0} J / 2\right) & 0 & 0 \\
\cosh \left(-\beta_{0} J / 2\right) & 0 & 0 & e^{-\beta_{0} J / 2}
\end{array}\right) .
$$

This is the smallest unit of the QTM shown in figure 8(b).

The initial blocks of the transfer matrix $\mathcal{T}^{A(M=1)}$ and $\mathcal{T}^{B(M=1)}$ in equation (49) are written as

$$
\begin{aligned}
& \mathcal{T}^{A(M=1)}\left(\sigma_{0, \tau_{0}}, \sigma_{0, \tau_{1}}\left|\sigma_{1, \tau_{0}}, \sigma_{1, \tau_{2}}\right| \sigma_{2, \tau_{1}}, \sigma_{2, \tau_{2}}\right) \\
& =\mathcal{T}^{B(M=1)}\left(\sigma_{0, \tau_{0}}, \sigma_{0, \tau_{1}}\left|\sigma_{1, \tau_{0}}, \sigma_{1, \tau_{2}}\right| \sigma_{2, \tau_{1}}, \sigma_{2, \tau_{2}}\right) \\
& =\sum_{\sigma_{1, \tau_{1}}} \hat{\Theta}\left(\sigma_{0, \tau_{0}} \sigma_{0, \tau_{1}} \mid \sigma_{1, \tau_{0}} \sigma_{1, \tau_{1}}\right) \hat{\Theta}\left(\sigma_{1, \tau_{1}} \sigma_{1, \tau_{2}} \mid \sigma_{2, \tau_{1}} \sigma_{2, \tau_{2}}\right)
\end{aligned}
$$

The QTM $\mathcal{T}^{(M=1)}$ is obtained from $\mathcal{T}^{A(M=1)}$ with the periodic boundary conditions in the $\beta$-direction $\sigma_{i, \tau_{2}}=\sigma_{i, \tau_{0}}$ :

$$
\begin{aligned}
& \mathcal{T}^{(M=1)}\left(\sigma_{0, \tau_{0}}, \sigma_{0, \tau_{1}} \mid \sigma_{2, \tau_{0}}, \sigma_{2, \tau_{1}}\right) \\
& =\sum_{\sigma_{1, \tau_{0}}} \sum_{\sigma_{1, \tau_{2}}} \sum_{\sigma_{2, \tau_{2}}} \mathcal{T}^{A(M=1)}\left(\sigma_{0, \tau_{0}}, \sigma_{0, \tau_{1}}\left|\sigma_{1, \tau_{0}}, \sigma_{1, \tau_{2}}\right| \sigma_{2, \tau_{1}}, \sigma_{2, \tau_{2}}\right) \prod_{i=1}^{2} \delta_{\sigma_{i, \tau_{2}} \sigma_{i, \tau_{0}}} .
\end{aligned}
$$

\subsection{Renormalization of the quantum transfer matrix}

The QTM at low temperature is obtained by extending the QTM in the $\beta$-direction. By using $\mathcal{T}^{A(M=1)}$ and $\mathcal{T}^{B(M=1)}$ defined in equation (75), we first calculate the QTM of $M=2$. This QTM corresponds to a high temperature of $T=1 /\left(M \beta_{0}\right)=1 /\left(2 \beta_{0}\right) \gg 1$ since we need to satisfy $\beta_{0}=\Delta \tau \ll 1$. We therefore decrease the temperature by iteratively increasing Trotter number $M$ as $T=1 /\left(M \beta_{0}\right) \rightarrow T^{\prime}=1 /\left((M+1) \beta_{0}\right)$ keeping $\beta_{0}$ fixed. In the following we describe detailed calculation for the extension of the QTM in the $\beta$-direction.

As is shown in figure 10 the $\mathcal{T}^{(M=2)}$ is written by $\mathcal{T}^{A(M=1)}$ and $\mathcal{T}^{B(M=1)}$ as

$$
\begin{aligned}
\mathcal{T}^{(M=2)}\left(\sigma_{0, \tau_{0}}, \sigma_{0, \tau_{1}}, \sigma_{0, \tau_{2}}, \sigma_{0, \tau_{3}} \mid \sigma_{2, \tau_{0}}, \sigma_{2, \tau_{1}}, \sigma_{2, \tau_{2}}, \sigma_{2, \tau_{3}}\right) & { }^{\sum_{\sigma_{1, \tau_{0}}, \sigma_{1, \tau_{2}}}} \mathcal{T}^{B(M=1)}\left(\sigma_{0, \tau_{2}}, \sigma_{0, \tau_{3}}\left|\sigma_{1, \tau_{2}}, \sigma_{1, \tau_{0}}\right| \sigma_{2, \tau_{3}}, \sigma_{2, \tau_{0}}\right) \\
& \times \mathcal{T}^{A(M=1)}\left(\sigma_{0, \tau_{0}}, \sigma_{0, \tau_{1}}\left|\sigma_{1, \tau_{0}}, \sigma_{1, \tau_{2}}\right| \sigma_{2, \tau_{1}}, \sigma_{2, \tau_{2}}\right) .
\end{aligned}
$$

We first calculate the maximum eigenvalue $\lambda_{\max }^{(M=2)}$ and the corresponding left and right eigenvectors, $V^{L(M=2)}\left(\sigma_{\tau_{0}}, \sigma_{\tau_{1}}, \sigma_{\tau_{2}}, \sigma_{\tau_{3}}\right)$ and $V^{R(M=2)}\left(\sigma_{\tau_{0}}, \sigma_{\tau_{1}}, \sigma_{\tau_{2}}, \sigma_{\tau_{3}}\right)$, by solving 
eigenvalue equations (56) and (57). We then calculate the density matrix $\rho$ for the restriction of the basis states of $\mathcal{T}^{A(M=1+1 / 2)}$ and $\mathcal{T}^{B(M=1+1 / 2)}$ :

$$
\begin{aligned}
& \rho\left(\sigma_{\tau_{1}}, \sigma_{\tau_{2}} \mid \sigma_{\tau_{1}}^{\prime}, \sigma_{\tau_{2}}^{\prime}\right) \\
& \quad=\sum_{\sigma_{\tau_{0}}, \sigma_{\tau_{3}}} V^{L(M=2)}\left(\sigma_{\tau_{0}}, \sigma_{\tau_{1}}, \sigma_{\tau_{2}}, \sigma_{\tau_{3}}\right) V^{R(M=2)}\left(\sigma_{\tau_{0}}, \sigma_{\tau_{1}}^{\prime}, \sigma_{\tau_{2}}^{\prime}, \sigma_{\tau_{3}}\right) \\
& \rho\left(\sigma_{\tau_{2}}, \sigma_{\tau_{3}} \mid \sigma_{\tau_{2}}^{\prime}, \sigma_{\tau_{3}}^{\prime}\right) \\
& \quad=\sum_{\sigma_{\tau_{0}}, \sigma_{\tau_{1}}} V^{L(M=2)}\left(\sigma_{\tau_{0}}, \sigma_{\tau_{1}}, \sigma_{\tau_{2}}, \sigma_{\tau_{3}}\right) V^{R(M=2)}\left(\sigma_{\tau_{0}}, \sigma_{\tau_{1}}, \sigma_{\tau_{2}}^{\prime}, \sigma_{\tau_{3}}^{\prime}\right) .
\end{aligned}
$$

The left and right eigenvectors $v_{\alpha}^{L}\left(\sigma_{\tau_{1}}, \sigma_{\tau_{2}}\right)$ and $v_{\alpha}^{R}\left(\sigma_{\tau_{1}}, \sigma_{\tau_{2}}\right)$ of the density matrix $\rho\left(\sigma_{\tau_{1}}, \sigma_{\tau_{2}} \mid \sigma_{\tau_{1}}^{\prime}, \sigma_{\tau_{2}}^{\prime}\right)$ are determined by the eigenvalue equations (59) and (60). $\mathcal{T}^{A(M=1+1 / 2)}$ in the new basis states is obtained by using the eigenvectors of the $m$ largest eigenvalues of the density matrix, $v_{\alpha_{0}}^{R}$ and $v_{\alpha_{2}}^{L}$ as

$$
\begin{aligned}
& \mathcal{T}^{A(M=1+1 / 2)}\left(\sigma_{0, \tau_{0}}, \alpha_{0, \tau_{1,2}}, \sigma_{0, \tau_{3}}\left|\sigma_{1, \tau_{0}}, \sigma_{1, \tau_{3}}\right| \alpha_{2, \tau_{1,2}}\right) \\
& =\sum_{\sigma_{0, \tau_{1}, \sigma_{0, \tau_{2}}}} \sum_{\sigma_{2, \tau_{1}, \sigma_{2, \tau_{2}}}} \sum_{\sigma_{1, \tau_{2}}} \mathcal{T}^{A(M=1)}\left(\sigma_{0, \tau_{0}}, \sigma_{0, \tau_{1}}\left|\sigma_{1, \tau_{0}}, \sigma_{1, \tau_{2}}\right| \sigma_{2, \tau_{1}}, \sigma_{2, \tau_{2}}\right) \\
& \quad \times \hat{\Theta}\left(\sigma_{0, \tau_{2}} \sigma_{0, \tau_{3}} \mid \sigma_{1, \tau_{2}} \sigma_{1, \tau_{3}}\right) v_{\alpha_{0}}^{R}\left(\sigma_{0, \tau_{1}}, \sigma_{0, \tau_{2}}\right) v_{\alpha_{2}}^{L}\left(\sigma_{2, \tau_{1}}, \sigma_{2, \tau_{2}}\right)
\end{aligned}
$$

where $\alpha_{0}$ and $\alpha_{2}$ corresponds to the new basis states defined by the $m$ largest eigenvalues of the density matrix. $\mathcal{T}^{B(M=1+1 / 2)}$ is similarly given by

$$
\begin{aligned}
& \mathcal{T}^{B(M=1+1 / 2)}\left(\alpha_{0, \tau_{2,3}}\left|\sigma_{1, \tau_{1}}, \sigma_{1, \tau_{4}}\right| \sigma_{2, \tau_{1}}, \alpha_{2, \tau_{2,3}}, \sigma_{2, \tau_{4}}\right) \\
& =\sum_{\sigma_{0, \tau_{2}, \sigma_{0, \tau_{3}}}} \sum_{\sigma_{2, \tau_{2}, \sigma_{2, \tau_{3}}}} \sum_{\sigma_{1, \tau_{2}}} \mathcal{T}^{B(M=1)}\left(\sigma_{0, \tau_{2}}, \sigma_{0, \tau_{3}}\left|\sigma_{1, \tau_{2}}, \sigma_{1, \tau_{4}}\right| \sigma_{2, \tau_{3}}, \sigma_{2, \tau_{4}}\right) \\
& \quad \times \hat{\Theta}\left(\sigma_{1, \tau_{1}} \sigma_{1, \tau_{2}} \mid \sigma_{2, \tau_{1}} \sigma_{2, \tau_{2}}\right) v_{\alpha_{0}}^{R}\left(\sigma_{0, \tau_{2}}, \sigma_{0, \tau_{3}}\right) v_{\alpha_{2}}^{L}\left(\sigma_{2, \tau_{2}}, \sigma_{2, \tau_{3}}\right) .
\end{aligned}
$$

The QTM of $M=3$ is obtained by using $\mathcal{T}^{A(M=1+1 / 2)}$ and $\mathcal{T}^{B(M=1+1 / 2)}$ as

$$
\begin{aligned}
& \mathcal{T}^{(M=3)}\left(\sigma_{0, \tau_{0}}, \alpha_{0, \tau_{1,2}}, \sigma_{0, \tau_{3}}, \alpha_{0, \tau_{4,5}} \mid \sigma_{2, \tau_{0}}, \alpha_{2, \tau_{1,2}}, \sigma_{2, \tau_{3}}, \alpha_{2, \tau_{4,5}}\right) \\
& =\sum_{\sigma_{1, \tau_{0}}, \sigma_{1, \tau_{3}}} \mathcal{T}^{B(M=1+1 / 2)}\left(\alpha_{0, \tau_{4,5}}\left|\sigma_{1, \tau_{3}}, \sigma_{1, \tau_{0}}\right| \sigma_{2, \tau_{3}}, \alpha_{2, \tau_{4,5}}, \sigma_{2, \tau_{0}}\right) \\
& \quad \times \mathcal{T}^{A(M=1+1 / 2)}\left(\sigma_{0, \tau_{0}}, \alpha_{0, \tau_{1,2}}, \sigma_{0, \tau_{3}}\left|\sigma_{1, \tau_{0}}, \sigma_{1, \tau_{3}}\right| \alpha_{2, \tau_{1,2}}\right) .
\end{aligned}
$$

The density matrices needed for the calculation of $\mathcal{T}^{A(M=2)}$ and $\mathcal{T}^{B(M=2)}$ are calculated from the eigenvectors of the QTM of $M=3$ :

$$
\begin{aligned}
& \rho\left(\alpha_{\tau_{1,2}}, \sigma_{\tau_{3}} \mid \alpha_{\tau_{1,2}}^{\prime}, \sigma_{\tau_{3}}^{\prime}\right) \\
& =\sum_{\sigma_{\tau_{0}}, \alpha_{\tau_{4}, 5}} V^{L(M=3)}\left(\sigma_{\tau_{0}}, \alpha_{\tau_{1,2}}, \sigma_{\tau_{3}}, \alpha_{\tau_{4,5}}\right) V^{R(M=3)}\left(\sigma_{\tau_{0}}, \alpha_{\tau_{1,2}}^{\prime}, \sigma_{\tau_{3}}^{\prime}, \alpha_{\tau_{4,5}}\right) \\
& \rho\left(\sigma_{\tau_{3}}, \alpha_{\tau_{4,5}} \mid \sigma_{\tau_{3}}^{\prime}, \alpha_{\tau_{4,5}}^{\prime}\right) \\
& =\sum_{\sigma_{\tau_{0}}, \alpha_{\tau_{1,2}}} V^{L(M=3)}\left(\sigma_{\tau_{0}}, \alpha_{\tau_{1,2}}, \sigma_{\tau_{3}}, \alpha_{\tau_{4,5}}\right) V^{R(M=3)}\left(\sigma_{\tau_{0}}, \alpha_{\tau_{1,2}}, \sigma_{\tau_{3}}^{\prime}, \alpha_{\tau_{4,5}}^{\prime}\right) .
\end{aligned}
$$

$\mathcal{T}^{A(M=2)}$ and $\mathcal{T}^{B(M=2)}$ are obtained by using the eigenvectors of the $m$ largest eigenvalues of the density matrix:

$$
\mathcal{T}^{A(M=2)}\left(\sigma_{0, \tau_{0}}, \alpha_{0, \tau_{1,2,3}}\left|\sigma_{1, \tau_{0}}, \sigma_{1, \tau_{4}}\right| \alpha_{2, \tau_{1,2,3}}, \sigma_{2, \tau_{4}}\right)
$$




$$
\begin{aligned}
& =\sum_{\alpha_{0, \tau_{1,2}}, \sigma_{0, \tau_{3}}} \sum_{\alpha_{2, \tau_{1,2}, \sigma_{2, \tau_{3}}}} \sum_{\sigma_{1, \tau_{3}}} \mathcal{T}^{A(M=1+1 / 2)}\left(\sigma_{0, \tau_{0}}, \alpha_{0, \tau_{1,2}}, \sigma_{0, \tau_{3}}\left|\sigma_{1, \tau_{0}}, \sigma_{1, \tau_{3}}\right| \alpha_{2, \tau_{1,2}}\right) \\
& \quad \times \hat{\Theta}\left(\sigma_{1, \tau_{3}} \sigma_{1, \tau_{4}} \mid \sigma_{2, \tau_{3}} \sigma_{2, \tau_{4}}\right) v_{\alpha_{0}}^{R}\left(\alpha_{0, \tau_{1,2}}, \sigma_{0, \tau_{3}}\right) v_{\alpha_{2}}^{L}\left(\alpha_{2, \tau_{1,2}}, \sigma_{2, \tau_{3}}\right) \\
& =\sum_{\sigma_{0, \tau_{3}}, \alpha_{0, \tau_{4}, 5}} \sum_{\sigma_{2, \tau_{3}, \alpha_{2, \tau_{4}, 5}}} \sum_{\sigma_{1, \tau_{3}}} \mathcal{T}^{B(M=2)}\left(\sigma_{0, \tau_{2}}, \alpha_{0, \tau_{3,4}, 5}\left|\sigma_{1, \tau_{2}}, \sigma_{1, \tau_{6}}\right| \alpha_{2, \tau_{3,4}, 5}, \sigma_{2, \tau_{6}}\right) \\
& \quad \times \hat{\Theta}\left(\sigma_{0, \tau_{2}} \sigma_{0, \tau_{3}} \mid \sigma_{1, \tau_{2}} \sigma_{1, \tau_{3}}\right) v_{\alpha_{0}}^{R}\left(\sigma_{0, \tau_{3}}, \alpha_{0, \tau_{4,5}}\right) v_{\alpha_{2}}^{L}\left(\sigma_{2, \tau_{3}}, \alpha_{2, \tau_{4,5}}\right) .
\end{aligned}
$$

$\mathcal{T}^{B(M=2)}$ shown in figure [10] and equation (55) is obtained by shifting the imaginary time indices as $\tau_{i} \rightarrow \tau_{i-2}$. Repeating the above procedures, we extend the QTM in the $\beta$-direction, and the QTM of large $M$ is obtained within a restricted number of basis states.

\subsection{Thermodynamic quantities}

The iterative extension of the QTM in the $\beta$-direction corresponds to a successive decrease in temperature, which is related to the Trotter number as $T=1 / \beta=1 /\left(\beta_{0} M\right)$. At each temperature, the free energy of the system par site is obtained from the maximum eigenvalue $\lambda_{\max }$

$$
F(T)=-\ln \lambda_{\max } /(2 \beta)
$$

The entropy $S$ is obtained by taking derivative of the free energy with respect to temperature

$$
S(T)=-\frac{\partial}{\partial T} F
$$

The specific heat $C$ is similarly obtained by taking second derivative of the free energy

$$
C(T)=-T \frac{\partial^{2}}{\partial T^{2}} F
$$

The magnetic susceptibility $\chi_{s}$ and the charge susceptibility $\chi_{c}$ are also calculated from the free energy at slightly different magnetic fields $h^{z}$ and chemical potentials $\mu$

$$
\begin{aligned}
& \chi_{s}=-\frac{\partial^{2}}{\partial h^{z^{2}}} F \\
& \chi_{c}=-\frac{\partial^{2}}{\partial \mu^{2}} F .
\end{aligned}
$$

The local quantities such as the magnetization and the density of electrons are obtained by using the eigenvectors $V^{R}$ and $V^{L}$ of $\lambda_{\max }$. The magnetization $\left\langle S_{0}^{z}\right\rangle$ and the density of electrons $\left\langle n_{0}\right\rangle$ are given by

$$
\begin{aligned}
& \left\langle S_{0}^{z}\right\rangle \equiv \operatorname{Tr}\left\{\mathrm{e}^{-\beta H} S_{0}^{z}\right\} / Z=\left\langle V^{L}\left|S_{0, \tau_{0}}^{z}\right| V^{R}\right\rangle \\
& \left\langle n_{0}\right\rangle \equiv \operatorname{Tr}\left\{\mathrm{e}^{-\beta H} n_{0}\right\} / Z=\left\langle V^{L}\left|n_{0, \tau_{0}}\right| V^{R}\right\rangle
\end{aligned}
$$




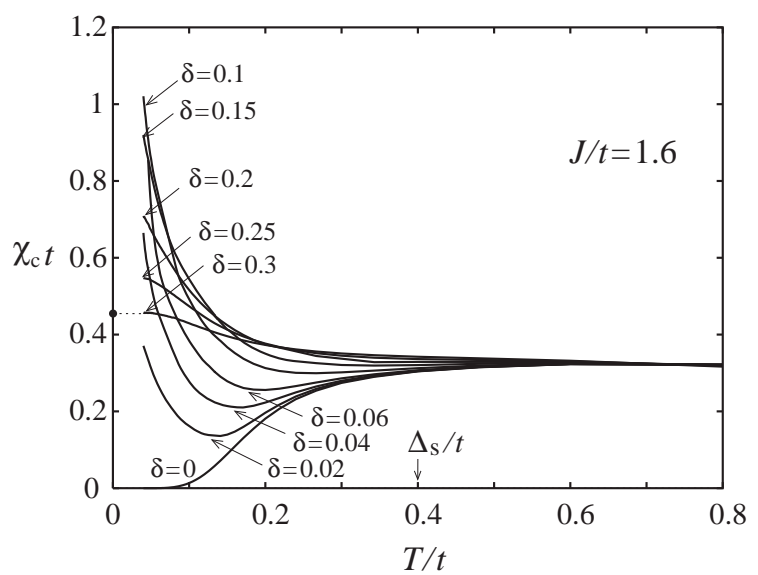

Figure 12. Charge susceptibility of the one-dimensional Kondo lattice model with hole doping $\delta$ in the insulating state at half-filling [11. The solid circle on the vertical axis represent the results obtained by the original zero-temperature DMRG method. $\Delta_{s}$ is the spin gap at $\delta=0 . J$ is the antiferromagnetic coupling between the conduction electrons and localized spins. $t$ is the hopping integral.

where $S_{0, \tau_{0}}^{z}$ is the operator at site $i=0$ and imaginary time $\tau_{0}$. The magnetic susceptibility $\chi_{s}$ is then obtained by applying a small magnetic field $h^{z}$

$$
\chi_{s}=\lim _{h^{z} \rightarrow 0}\left\langle S_{0}^{z}\right\rangle / h^{z} .
$$

Similarly, the charge susceptibility $\chi_{c}$ is obtained by changing chemical potential $\mu$

$$
\chi_{c}=\lim _{\Delta \mu \rightarrow 0}\left(\left\langle n_{0}\right\rangle_{\mu+\Delta \mu}-\left\langle n_{0}\right\rangle_{\mu}\right) / \Delta \mu .
$$

As a typical example, the charge susceptibility of the one-dimensional Kondo lattice model calculated by the present method is shown in figure 12.

The nearest-neighbor and next-nearest neighbor correlations are also obtained by using the eigenvectors $V^{R}$ and $V^{L}$ and the QTM $\mathcal{T}$

$$
\begin{aligned}
& \left\langle S_{0}^{z} S_{1}^{z}\right\rangle \equiv \operatorname{Tr}\left\{\mathrm{e}^{-\beta H} S_{0}^{z} S_{1}^{z}\right\} / Z=\left\langle V^{L}\left|S_{0, \tau_{0}}^{z} \mathcal{T} S_{1, \tau_{0}}^{z}\right| V^{R}\right\rangle / \lambda_{\max } \\
& \left\langle S_{0}^{z} S_{2}^{z}\right\rangle \equiv \operatorname{Tr}\left\{\mathrm{e}^{-\beta H} S_{0}^{z} S_{2}^{z}\right\} / Z=\left\langle V^{L}\left|S_{0, \tau_{0}}^{z} \mathcal{T} S_{2, \tau_{0}}^{z}\right| V^{R}\right\rangle / \lambda_{\max }
\end{aligned}
$$

In equation (96) we have used $S_{i}^{z}$ commute with the local Hamiltonian $h_{i, i+1}$. The internal energy of the system is calculated from the above expectation values and we can calculate the specific heat $C$ by taking derivative of the internal energy $E$ with respect to temperature

$$
C(T)=\frac{\partial}{\partial T} E
$$

In order to confirm the accuracy of the results, we need to increase the number of basis states $m$ kept in each block and the Trotter number $M$ of the QTM. The error caused by finite $M$ is usually scaled by $M^{-2}$, and the correct value in the limit of $M \rightarrow \infty$ is obtained by the linear extrapolation with respect to $M^{-2}$. The error caused by finite $m$ does not follow a simple scaling form in general. Nevertheless, the truncation error in the norm of the eigenvectors $\left\langle V^{L} \mid V^{R}\right\rangle$ is given by $1-\sum_{k=1}^{m} w^{k}$, where $w^{k}$ is the 


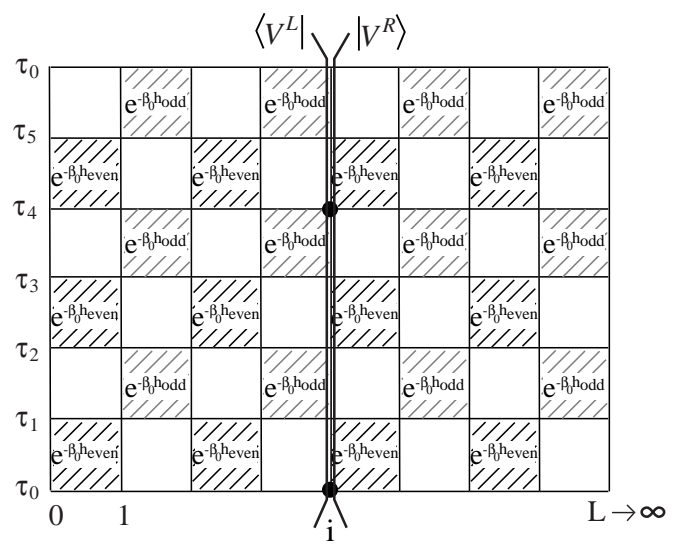

Figure 13. Schematic diagram of the calculation of imaginary-time correlation functions with the eigenvectors $\left\langle V^{L}\right|$ and $\left|V^{R}\right\rangle$.

$k$ th largest eigenvalue of the density matrix. The correction for the thermodynamic quantities will be estimated from the truncation error.

\subsection{Dynamic correlation function}

The Green's functions and dynamic correlation functions are obtained from the imaginary time Green's functions and correlation functions through analytical continuation to the real frequency axis[12]. The imaginary time Green's function is calculated from the eigenvectors of the QTM as

$$
\begin{aligned}
G\left(\tau_{2 j}\right) & \equiv-\operatorname{Tr}\left\{\mathrm{e}^{-\beta H} c_{i \sigma}\left(\tau_{2 j}\right) c_{i \sigma}^{\dagger}(0)\right\} / Z \\
& =-\left\langle V^{L}\left|c_{i \sigma}\left(\tau_{2 j}\right) c_{i \sigma}^{\dagger}(0)\right| V^{R}\right\rangle
\end{aligned}
$$

where $\left\langle V^{L}\right|$ and $\left|V^{R}\right\rangle$ are eigenvectors of the largest eigenvalue $\lambda_{\max }$, and $\tau_{2 j}=j(\beta / M)$. The local dynamic correlation function $\chi_{A B}\left(\tau_{2 j}\right)$ on the $\beta$-axis is also obtained by

$$
\begin{aligned}
\chi_{A B}\left(\tau_{2 j}\right) & \equiv \operatorname{Tr}\left\{\mathrm{e}^{-\beta H} A_{i}\left(\tau_{2 j}\right) B_{i}(0)\right\} / Z \\
& =\left\langle V^{L}\left|A_{i}\left(\tau_{2 j}\right) B_{i}(0)\right| V^{R}\right\rangle .
\end{aligned}
$$

The frequency dependence of the imaginary time Green's function and correlation functions are obtained by Fourier transformation along the imaginary axis

$$
\begin{aligned}
& G\left(i \omega_{n}\right)=\frac{\beta}{M} \sum_{j=0}^{M} \mathrm{e}^{i \omega_{n} \tau_{2 j}} G\left(\tau_{2 j}\right) \\
& \chi_{A B}\left(i \omega_{n}\right)=\frac{\beta}{M} \sum_{j=0}^{M} \mathrm{e}^{i \omega_{n} \tau_{2 j}} \chi_{A B}\left(\tau_{2 j}\right)
\end{aligned}
$$

where $\omega_{n}$ is the Matsubara frequency that is $\pi(2 n+1) / \beta$ for fermionic operators and $2 \pi n / \beta$ for bosonic operators.

The real frequency Green's functions and dynamic susceptibility are obtained by the analytical continuation to the real frequency axis. The Padé approximations or the 


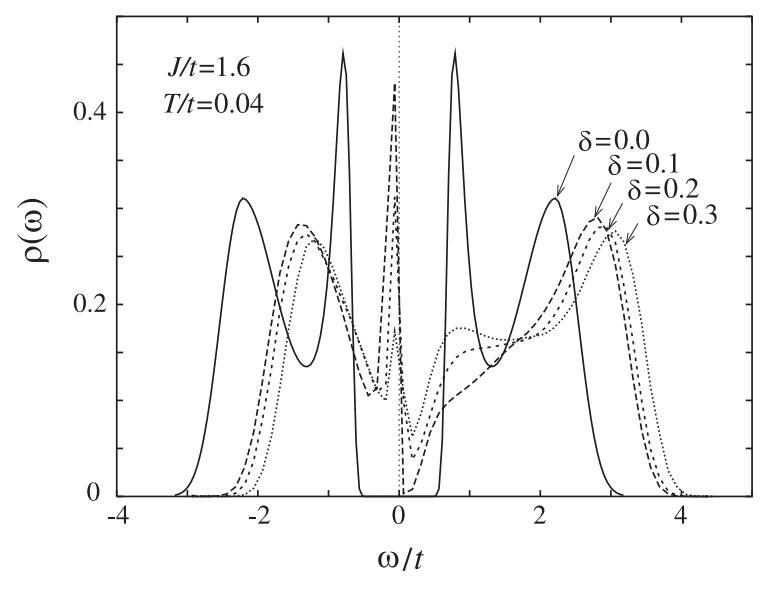

Figure 14. Quasi-particle density of states calculated in the one-dimensional Kondo lattice model with hole doping $\delta$ in the insulating state at half-filling [17].

maximum entropy method [53, 54] are used for this calculation. The Padé approximation is a fitting of $G\left(i \omega_{n}\right)$ or $\chi_{A B}\left(i \omega_{n}\right)$ by rational functions of frequency $i \omega_{n}$, which are analytically continued to the real axis by $i \omega_{n} \rightarrow \omega+i \delta$.

The maximum entropy method is based on the spectral representations

$$
\begin{aligned}
& G(\tau)=\int_{-\infty}^{\infty} \rho(\omega) \frac{\mathrm{e}^{-\tau \omega}}{1+\mathrm{e}^{-\beta \omega}} d \omega \\
& \chi_{A B}(\tau)=\int_{-\infty}^{\infty} \frac{1}{\pi} \operatorname{Im} \chi_{A B}(\omega) \frac{\mathrm{e}^{-\tau \omega}}{1-\mathrm{e}^{-\beta \omega}} d \omega
\end{aligned}
$$

where $\rho(\omega)=-\frac{\operatorname{Im}}{\pi} G(\omega+i \delta)$ is the density of state. This method finds the best $\rho(\omega)$ and $\chi_{A B}(\omega)$ that reproduce $G(\tau)$ and $\chi_{A B}(\tau)$.

The dynamic structure factor $S_{A B}(\omega)$ is obtained from the imaginary part of $\chi_{A B}(\omega)$ through the fluctuation dissipation theorem

$$
\operatorname{Im} \chi_{A B}(\omega)=\pi\left(1-\mathrm{e}^{-\beta \omega}\right) S_{A B}(\omega) .
$$

\subsection{Finite-T DMRG with fixed Trotter number}

Reliable analytical continuation is only possible when we have accurate imaginary time correlation functions. In order to obtain correct dynamic properties, it is inevitable to use the finite system algorithm of the DMRG and to refine the eigenvectors $V^{R}$ and $V^{L}$ of the QTM. In the finite system algorithm we reconstruct the blocks $\mathcal{T}^{A}$ and $\mathcal{T}^{B}$ by keeping the Trotter number fixed. We first extend $\mathcal{T}^{A}$ until $\mathcal{T}^{B}$ is reduced down to the initial block of $\mathcal{T}^{B(M=1)}$, and then extend $\mathcal{T}^{B}$ to refine its basis states. We repeat such sweeps until the eigenvectors $V^{R}$ and $V^{L}$ are converged. Figure 14 shows the quasiparticle density of states calculated from the eigenvectors obtained by the finite system algorithm of the DMRG. In this figure we find the closing of the gap at the Fermi level $\omega=0$ with the hole doping $\delta$ in the Kondo insulator [17]. 


\subsection{Conserved quantities of the quantum transfer matrix}

The eigenvalues of the non-Hermitian matrix are not restricted to a real number. This fact sometimes causes uncontrolled instability generated from small numerical errors. In order to suppress such numerical instability and to keep the accuracy of the calculation, a precise diagonalization of the non-Hermitian matrix is needed. For this purpose, it is efficient to reduce the Hilbert space by using the conserved quantities of the QTM, which are related to the conservation law in the system. For example, $S_{i}^{z}+S_{i+1}^{z}$ in the Heisenberg model is conserved at any imaginary time evolution generated by $h_{i, i+1}$. This means the following equation is satisfied for each imaginary time evolution from $\tau_{2 k+i}$ to $\tau_{2 k+i+1}$ :

$$
S_{i, \tau_{2 k+i}}^{z}+S_{i+1, \tau_{2 k+i}}^{z}=S_{i, \tau_{2 k+i+1}}^{z}+S_{i+1, \tau_{2 k+i+1}}^{z} .
$$

This equation is translated to

$$
S_{i, \tau_{2 k+i}}^{z}-S_{i, \tau_{2 k+i+1}}^{z}=-S_{i+1, \tau_{2 k+i}}^{z}+S_{i+1, \tau_{2 k+i+1}}^{z}
$$

and the following equation is obtained after the summation over $k$

$$
\sum_{j=0}^{2 M-1}(-1)^{j+i} S_{i, \tau_{j}}^{z}=\sum_{j=0}^{2 M-1}(-1)^{j+i+1} S_{i+1, \tau_{j}}^{z} .
$$

This result clearly shows that $Q_{i}$ defined by

$$
Q_{i}=\sum_{j=0}^{2 M-1}(-1)^{j+i} S_{i, \tau_{j}}^{z}
$$

is a conserved quantity of the QTM[51, 52].

Similar calculations are possible for charge degrees of freedom. For example,

$$
P_{i}=\sum_{j=0}^{2 M-1}(-1)^{j+i} n_{i, \tau_{j}}
$$

is a conserved quantity of the QTM, when $h_{i, i+1}$ preserves number of electrons $n_{i}+n_{i+1}$.

By using these conserved quantities, we can classify the basis states of the QTM. Since there is no matrix element between different subspaces classified by $Q_{i}$ and $P_{i}$, the QTM becomes block diagonal. The maximum eigenvalue $\lambda_{\max }$ of the QTM is then determined by calculating the largest eigenvalue in each subblock. The $\lambda_{\max }$ of the QTM is obtained in the subblock of $Q_{i}=P_{i}=0$ in most cases. The density matrix calculated from the eigenvectors of the QTM is also block diagonal, and we need only diagonalize the density matrix within each subblock. The reduction of matrix dimension improves the accuracy of the diagonalization and stabilizes the calculation.

\subsection{Complex eigenvalues of density matrix}

We finally comment on the problem of complex eigenvalues. This is caused by the non-Hermiticity of the density matrix, which allows appearance of complex eigenvalues. These are usually generated by the artificial truncation of basis states, and the imaginary parts of the eigenvalues are typically much smaller than their real parts. The complex 
eigenvalues always appear with their complex conjugate, and their eigenvectors are mutually complex conjugate of the other one. Thus we can define two real vectors, which span the Hilbert space generated by the eigenvectors of the two complex eigenvalues, from the real and the imaginary parts of the eigenvectors, provided that the imaginary parts of the eigenvalues are sufficiently small compared with its real part. After checking the dual orthogonality of the left and right basis states $\langle i \mid j\rangle=\delta_{i j}$, we can continue the DMRG calculation using the basis states defined by the real vectors.

\section{Application to two-dimensional systems}

Electrons in two-dimensional systems exhibit various interesting phenomena. Although their properties have been extensively studied for a long time, many unresolved questions are still remaining. In order to study the ground-state and thermodynamic properties of the system, the quantum Monte Carlo method and the exact diagonalizations have been used. However, when the electron density is away from particle hole symmetric point, the negative sign problem arises in quantum Monte Carlo simulations. Although the exact diagonalization method provides rigorous results, the number of electrons in the system is limited to small numbers. Thus, we need a new reliable method for two-dimensional electron systems away from half-filling, and various applications of the DMRG to twodimensional systems have been considered. Most of the applications use mappings on to effective one-dimensional systems. However, the mapping is not uniquely determined and many long-range interactions appear, depending on the system. We thus need to find appropriate mapping specific to each model.

\subsection{Mapping to the one-dimensional lattice model}

In this section we consider two-dimensional electrons in a magnetic field. In twodimensional systems under a perpendicular magnetic field, the kinetic energy of the electrons is completely quenched and the remaining macroscopic degeneracy is lifted by Coulomb interaction. Such an interesting system is realized in quantum Hall systems, where various electronic states have been observed. Here we describe how the DMRG method is applied to quantum Hall systems 34, 37.

In order to represent the Hamiltonian in matrix form, we first define one-particle states $\Psi_{N X}(x, y)$ of two-dimensional electrons. We use the eigenstates of free electrons in perpendicular magnetic field and represent the wavefunction in the Landau gauge. The one-particle states are represented by the following wavefunctions and each state is uniquely specified by only two numbers $N$ and $X$ :

$$
\Psi_{N X}=C_{N} \exp \left[i k_{y} y-\frac{(x-X)^{2}}{2 \ell^{2}}\right] H_{N}\left[\frac{x-X}{\ell}\right]
$$

where $N$ is the Landau level index and $X$ is the $x$-component of the guiding center coordinates of an electron in cyclotron motion. $H_{N}$ are Hermite polynomials and $C_{N}$ is 
the normalization constant. $\ell=(\hbar c / e H)^{1 / 2}$ is the magnetic length, which is the lengthscale of the system. The guiding center $X$ is related to the momentum $k_{y}$ through $X=k_{y} \ell^{2}$. Since $k_{y}$ is discretized under the periodic boundary conditions, the guiding center $X$ takes only discrete values

$$
X_{n}=2 \pi \ell^{2} n / L_{y}
$$

where $L_{y}$ is the length of the unit cell in the $y$-direction. After we have fixed the Landau level index, each one-particle state is uniquely specified by the guiding center $X_{n}$. Thus the Hamiltonian is mapped on to an effective one-dimensional lattice model.

The ground state of two-dimensional electrons in a perpendicular magnetic field is determined only by the Coulomb interaction

$$
V(r)=\frac{e^{2}}{\epsilon r}
$$

The Coulomb interaction generates correlations between the electrons and stabilize various electronic states depending on the filling $\nu$ of Landau levels. When the magnetic field is strong enough so that the Landau level splitting is sufficiently large compared with the typical Coulomb interaction $e^{2} /(\epsilon \ell)$, the electrons in fully occupied Landau levels are inert and the ground state is determined only by the electrons in the top most partially filled Landau level.

The Hamiltonian is then written by

$$
H=S \sum_{n} c_{n}^{\dagger} c_{n}+\frac{1}{2} \sum_{n_{1}} \sum_{n_{2}} \sum_{n_{3}} \sum_{n_{4}} A_{n_{1} n_{2} n_{3} n_{4}} c_{n_{1}}^{\dagger} c_{n_{2}}^{\dagger} c_{n_{3}} c_{n_{4}}
$$

where we have imposed periodic boundary conditions in both $x$ - and $y$-directions, and $S$ is the classical Coulomb energy of Wigner crystal with a rectangular unit cell of $L_{x} \times L_{y}[55] . c_{n}^{\dagger}$ is the creation operator of the electron represented by the wavefunction defined in equation (111) with $X=X_{n}$. $A_{n_{1} n_{2} n_{3} n_{4}}$ are the matrix elements of the Coulomb interaction defined by

$$
\begin{aligned}
& A_{n_{1} n_{2} n_{3} n_{4}}=\delta_{n_{1}+n_{2}, n_{3}+n_{4}}^{\prime} \frac{1}{L_{x} L_{y}} \sum_{\mathbf{q}} \delta_{n_{1}-n_{4}, q_{y} L_{y} / 2 \pi}^{\prime} \frac{2 \pi e^{2}}{\epsilon q} \\
& \times\left[L_{N}\left(q^{2} \ell^{2} / 2\right)\right]^{2} \exp \left[-\frac{q^{2} \ell^{2}}{2}-i\left(n_{1}-n_{3}\right) \frac{q_{x} L_{x}}{M}\right]
\end{aligned}
$$

where $L_{N}(x)$ are Laguerre polynomials with $N$ being the Landau level index [56]. $\delta_{n_{1}, n_{2}}^{\prime}=1$ when $n_{1}=n_{2}(\bmod M)$ with $M$ being the number of one-particle states in the unit cell, which is given by the area of the unit cell $2 \pi M \ell^{2}=L_{x} L_{y}$.

In order to obtain the ground-state wavefunction we apply the DMRG method described in section 2. We start from a small-size system consisting of only four oneparticle states whose indices $n$ are $1,2, M-1$, and $M$, and we calculate the ground-state wavefunction. We then construct the left block containing local states of $n=1$ and 2 , and the right block containing $n=M-1$ and $M$ from the eigenvectors of the density matrices which are calculated from the ground-state wavefunction. We then add two local states $n=3$ and $M-2$ between the two blocks and repeat the above procedure until 


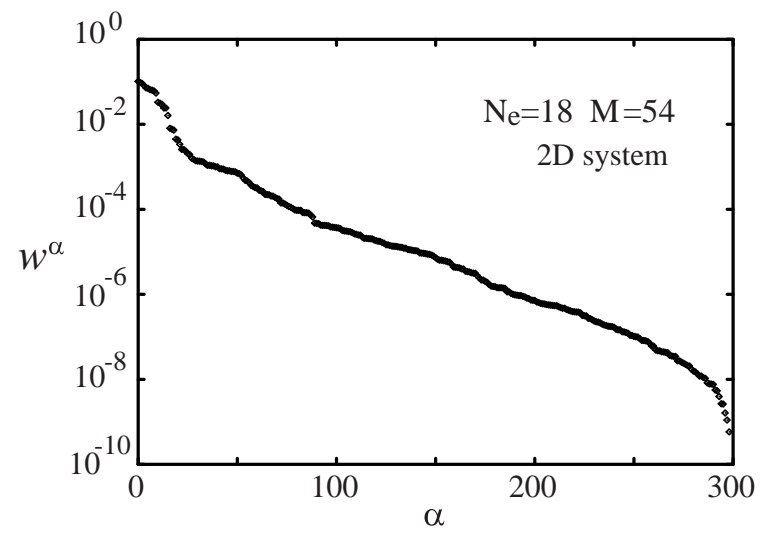

Figure 15. Eigenvalues of the density matrix for two-dimensional system with 18 electrons in 54 local orbitals.

$M$ local states are included in the system. We then apply the finite system algorithm of the DMRG to refine the ground-state wavefunction. After we obtain the convergence, we calculate correlation functions.

The ground-state pair correlation function $g(\mathbf{r})$ in guiding center coordinates is defined by

$$
g(\mathbf{r})=\frac{L_{x} L_{y}}{N_{e}\left(N_{e}-1\right)}\left\langle\Psi\left|\sum_{i \neq j} \delta\left(\mathbf{r}-\mathbf{R}_{i}+\mathbf{R}_{j}\right)\right| \Psi\right\rangle
$$

where $\mathbf{R}_{i}$ is the guiding center coordinate of the $i$ th electron, and it is calculated from the following equation

$$
\begin{aligned}
g(\mathbf{r})=\frac{1}{N_{e}\left(N_{e}-1\right)} \sum_{\mathbf{q}} \sum_{n_{1}, n_{2}, n_{3}, n_{4}} & \exp \left[i \mathbf{q} \cdot \mathbf{r}-\frac{q^{2} \ell^{2}}{2}-i\left(n_{1}-n_{3}\right) \frac{q_{x} L_{x}}{M}\right] \\
\times & \delta_{n_{1}-n_{4}, q_{y} L_{y} / 2 \pi}^{\prime}\left\langle\Psi\left|c_{n_{1}}^{\dagger} c_{n_{2}}^{\dagger} c_{n_{3}} c_{n_{4}}\right| \Psi\right\rangle
\end{aligned}
$$

where $\Psi$ is the ground state and $N_{e}$ is the total number of electrons.

The accuracy of the results depends on the distribution of eigenvalues of the density matrix. A typical example of the eigenvalues of the density matrix for system of $M=54$ with 18 electrons is shown in figure 15, which shows an exponential decrease of

eigenvalues $w^{\alpha}$. In this case the accuracy of $10^{-4}$ is obtained by keeping only 200 states in each block.

\subsection{Conserved quantities in magnetic field}

The Hamiltonian written in equation (114) conserves center of mass of electrons $G=$ $\sum_{i}\left(X_{n}\right)_{i}$, whose origin is the conservation of total $y$-momentum $\sum_{i}\left(k_{y}\right)_{i}=\sum_{i}\left(X_{n}\right)_{i} / \ell^{2}$. All the eigenstates of the Hamiltonian and the basis states of the blocks are classified by the quantum numbers, $N_{e}$ and $G$. These conservation laws restrict the basis states of the ground state, and the complete basis states of the extended system are not generally constructed from the eigenstates of the density matrix calculated from the groundstate wavefunction which has fixed numbers $N_{e}$ and $G$. Although this unexpected 


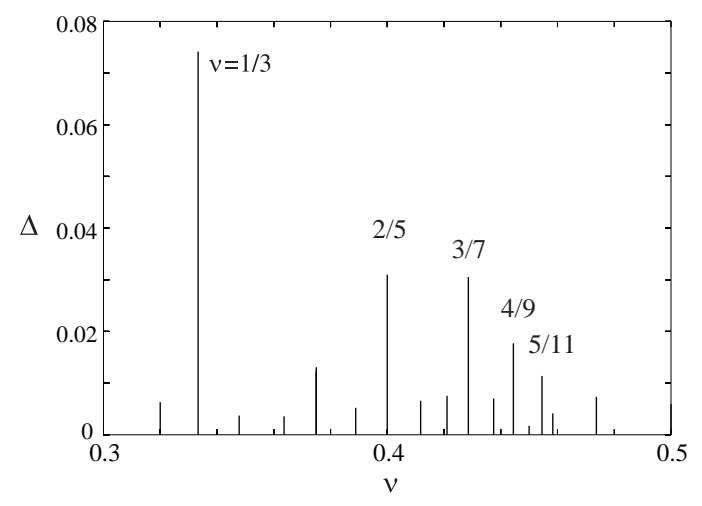

Figure 16. The lowest excitation gap at various $\nu$ in the lowest Landau level. Relatively large excitation gap is obtained at fractional fillings $\nu=n /(2 n+1)$. The excitation gap is in units of $e^{2} /(\epsilon \ell)$.

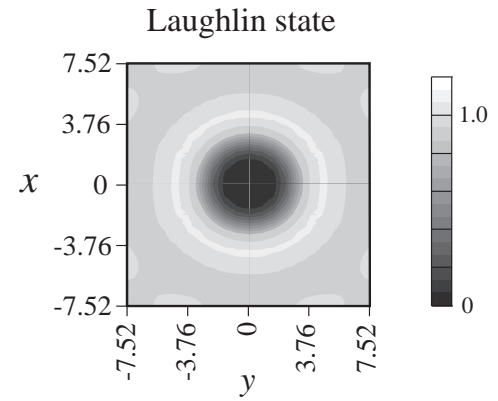

Figure 17. Pair correlation function $g(\mathbf{r})$ at $\nu=1 / 3$ in the lowest Landau level. The length is in units of $\ell$.

truncation is corrected by reconstructing the blocks using the finite system algorithm of the DMRG, we need to include additional basis states in the extended blocks to accelerate convergence in the infinite system algorithm of the DMRG.

\subsection{Diverse ground states in a magnetic field}

Here we present diverse ground states obtained by the DMRG method applied to the quantum Hall systems. In the limit of a strong magnetic field, the electrons occupy only the lowest Landau level $N=0$. In this limit, fractional quantum Hall effect (FQHE) has been observed at various fillings [57]. The FQHE state is characterized by incompressible liquid with a finite excitation gap 58 . The presence of the FQHE is confirmed by the DMRG calculations, where a relatively large excitation gap is obtained at various fillings between $\nu=1 / 2$ and 3/10 [37] as shown in figure 16] We clearly find large excitation gaps at fractional fillings $\nu=1 / 3,2 / 5,3 / 7,4 / 9$ and $5 / 11$, which correspond to primary series of the FQHE at $\nu=n /(2 n+1)$. The pair correlation function at $\nu=1 / 3$ is presented in figure 17, which shows a circularly symmetric liquid state consistent with the Laughlin state 58 . 
(a) Wigner crystal

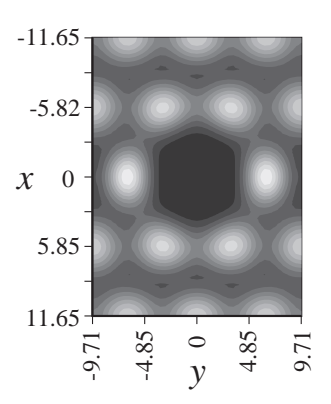

(b) two-electron

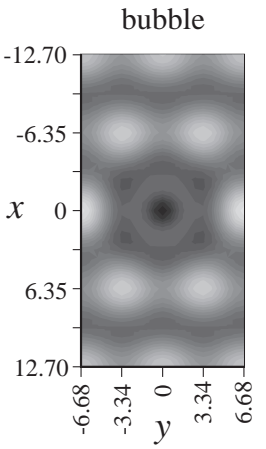

(c) stripe

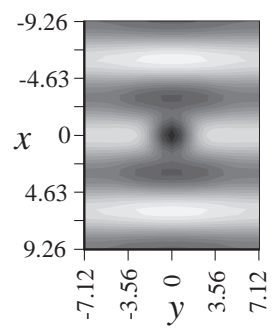

Figure 18. Pair correlation functions $g(\mathbf{r})$ in guiding center coordinates. (a) The Wigner crystal realized in an excited state at $\nu=1 / 6$ in the lowest Landau level. The number of electrons in the unit cell $N_{e}$ is 12 . (b) Two-electron bubble state at $\nu=8 / 27$ in the third lowest Landau level. $N_{e}=16$. (c) Stripe state at $\nu=3 / 7$ in the third lowest Landau level. $N_{e}=18$.

In the limit of low filling $\nu \rightarrow 0$, mean separation between the electrons becomes much longer than the typical length-scale of the one-particle wavefunction. In this limit the quantum fluctuations are not important and electrons behave as classical point particles, whose ground state is the Wigner crystal. The formation of the Wigner crystal is also confirmed by the DMRG calculations at low fillings as shown in figure 18 (a). The $\nu$ dependence of the low-energy spectrum shows that the first-order transition to the Wigner crystal occurs at $\nu \sim 1 / 7$.

With decreasing magnetic field, electrons occupy higher Landau levels. In high Landau levels, the one-particle wavefunction extends over space, generating effective long-range exchange interactions between the electrons. The long-range interaction prefers CDW ground states and Hartree-Fock calculations predict various CDW states called stripe and bubble[59]. These CDW states are confirmed by the DMRG calculations as shown in figures [18 (b) and (c). Although the CDW structures are similar to those obtained in the Hartree-Fock calculations, the ground-state energy and the phase diagram is significantly different 34. The DMRG results are consistent with recent experiments 60 , and the discrepancy is due to the quantum fluctuations neglected in the Hartree-Fock calculations.

\section{Summary}

In this topical review, we have reviewed the DMRG method and its applications to finite temperature and two-dimensional systems. We first briefly reviewed the original DMRG method, which enables us to calculate the ground-state wavefunctions and lowenergy excitations of large-size systems with controlled accuracy. The essential idea of the DMRG is the restriction of basis states using the eigenvectors of the density matrix calculated from the ground-state wavefunction. The truncation error of the wavefunction is estimated from the eigenvalues of the density matrix and we can extend the size of 
system with controlled accuracy.

This idea of the DMRG was applied to the QTM in one-dimensional systems, and a reliable method for the calculation of thermodynamic quantities at finite temperatures has been formulated. In this way the thermodynamic quantities are directly obtained from the maximum eigenvalue of the QTM, and temperature dependence is systematically obtained by extending the QTM in the $\beta$-direction. The dynamic correlation functions are calculated from the eigenvectors of the QTM with the analytical continuation to the real frequency axis.

We have also shown that the DMRG method is reliably applied to two-dimensional quantum systems in a magnetic field. We use the eigenstates of free electrons in the Landau gauge as initial basis states of two-dimensional electrons, and map the system

on to an effective one-dimensional lattice model with long-range interactions. It has been shown that this method is successfully applied to quantum Hall systems and the existence of various ground states has been confirmed.

\section{Acknowledgments}

The author thanks Professor Daijiro Yoshioka for suggesting the application of the DMRG to quantum Hall systems. The present work is financially supported by Grantin-Aid from MEXT, Japan.

\section{References}

[1] White S R 1992 Phys. Rev. Lett. 692863

[2] White S R 1993 Phys. Rev. B 4810345

[3] Nishino T 1995 J. Phys. Soc. Japan 643598

[4] Bursill R J, Xiang T and Gehring G A 1996 J. Phys.: Condens. Matter 8 L583

[5] Wang X and Xiang T 1997 Phys. Rev. B 565061

[6] Shibata N 1997 J. Phys. Soc. Japan 662221

[7] Xiang T 1998 Phys. Rev. B 589142

[8] Shibata N, Ammon B, Troyer M, Sigrist M, and Ueda K J. Phys. Soc. Japan 1998671086

[9] Maisinger K and Schollwöck U, 1999 Phys. Rev. Lett. 81445

[10] Ammon B, Troyer M, Rice T M and Shibata N 1999 Phys. Rev. Lett. 823855

[11] Shibata N and Tsunetsugu H 1999 J. Phys. Soc. Japan 68744

[12] Mutou T, Shibata N and Ueda K 1999 Phys. Rev. Lett. 81 4939; 1999 ibid. 823727

[13] Shibata N and Ueda K 1999 J. Phys.: Condens. Matter 11 R1; 1999 ibid. 114289

[14] Naef F, Wang X, Zotos X and Linden W von der 1999 Phys. Rev. B 60359

[15] Klümper A, Raupach R and Schönfeld F 1999 Phys. Rev. B 59 3612; 2000 Euro. Phys. J. B 1751

[16] Rommer S and Eggert S 1999 Phys. Rev. B 596301

[17] Shibata N and Tsunetsugu H 1999 J. Phys. Soc. Japan 683138

[18] Naef F and Wang X 2000 Phys. Rev. Lett. 841320

[19] Maeshima N and Okunishi K 2000 Phys. Rev. B 62934

[20] Wang X and Yu L 2000 Phys. Rev. Lett. 845399

[21] Ammon B and Imada M 2000 Phys. Rev. Lett. 851056

[22] Ammon B and Imada M 2000 J. Phys. Soc. Japan 691946

[23] Ammon B and Imada M 2001 J. Phys. Soc. Japan 70547

[24] Shibata N and Ueda K 2001 J. Phys. Soc. Japan 703690 
[25] Iwasa K, Kohgi M, Gukasov A, Mignot J -M, Shibata N, Ochiai A, Aoki H and Suzuki T 2002 Phys. Rev. B 65052408

[26] Sirker J and Klümper A 2002 Europhys. Lett. 60262

[27] Sirker J and Klümper A 2002 Phys. Rev. B 66245102

[28] Maruyama I, Shibata N and Ueda K 2002 Phys. Rev. B 65174421

[29] Sirker J and Khaliullin G 2003 Phys. Rev. B 67100408

[30] White S R 1996 Phys. Rev. Lett. 773633

[31] White S R and Scalapino D J 1998 Phys. Rev. Lett. 801272

[32] White S R and Scalapino D J 1999 Phys. Rev. B 60 R753

[33] White S R and Scalapino D J 2000 Phys. Rev. B 616320

[34] Shibata N and Yoshioka D 2001 Phys. Rev. Lett. 865755

[35] Yoshioka D and Shibata N 2002 Physica E 1243

[36] Xiang T 2001 Phys. Rev. B 64104414

[37] Shibata N and Yoshioka D 2003 J. Phys. Soc. Japan 72664

[38] Xiang T 1996 Phys. Rev. B 53 R10445

[39] Nishimoto S, Jeckelmann E, Gebhard F and Noack R M 2002 Phys. Rev. B 65165114

[40] Nishino T and Okunishi K 1996 J. Phys. Soc. Japan 65 891; 1997 J. Phys. Soc. Japan 663040

[41] Nishino T and Okunishi K 1998 J. Phys. Soc. Japan 673066

[42] Kemper A, Gendiar A, Nishino T, Schadschneider A and Zittartz J 2003 J. Phys. A: Math. Gen. 3629

[43] Maeshima N, Hieida Y, Akutsu Y, Nishino T and Okunishi K 2001 Phys. Rev. E 64016705

[44] Chung M C and Peschel I 2000 Phys. Rev. B 624191

[45] Peschel I, Wang X, Kaulke M and Hallberg K (eds) 1998 Density-Matrix Renormalization (Heidelberg: Springer)

[46] Betsuyaku H 1984 Phys. Rev. Lett. 53629

[47] Betsuyaku H 1985 Prog. Theor. Phys. 73319

[48] Trotter H F 1959 Proc. Amer. Math. Soc. 10545

[49] Suzuki M 1976 Commun. Math. Phys. 51183

[50] Suzuki M 1985 Phys. Rev. B 312957

[51] Koma T 1987 Prog. Theor. Phys. 78 1213; 1989 ibid. 81783

[52] Nomura K and Yamada M 1991 Phys. Rev. B 438217

[53] Silver R N, Sivia D S and Gubernatis J E 1990 Phys. Rev. B 412380

[54] Gubernatis J E, Jarrell M and Silver R N 1991 Phys. Rev. B 446011

[55] Bonsall L and Maradudin A 1977 Phys. Rev. B 151959

[56] Yoshioka D 1984 Phys. Rev. B 296833

[57] Du R R, Stormer H L, Tsui D C, Pfeiffer L N and West K W 1993 Phys. Rev. Lett. 702944

[58] Laughlin R B 1983 Phys. Rev. Lett. 501395

[59] Koulakov A A, Fogler M M and Shklovskii B I 1996 Phys. Rev. Lett. 76499

[60] Lilly M P, Cooper K B, Eisenstein J P, Pfeiffer L N and West K W 1999 Phys. Rev. Lett. 82394 